\title{
A SHORTEST PATH ALGORITHM FOR REAL-WEIGHTED UNDIRECTED GRAPHS*
}

\author{
SETH PETTIE ${ }^{\dagger}$ AND VIJAYA RAMACHANDRAN ${ }^{\ddagger}$
}

\begin{abstract}
We present a new scheme for computing shortest paths on real-weighted undirected graphs in the fundamental comparison-addition model. In an efficient preprocessing phase our algorithm creates a linear-size structure that facilitates single-source shortest path computations in $O(m \log \alpha)$ time, where $\alpha=\alpha(m, n)$ is the very slowly growing inverse-Ackermann function, $m$ the number of edges, and $n$ the number of vertices. As special cases our algorithm implies new bounds on both the all-pairs and single-source shortest paths problems. We solve the all-pairs problem in $O(m n \log \alpha(m, n))$ time and, if the ratio between the maximum and minimum edge lengths is bounded by $n^{(\log n)^{O(1)}}$, we can solve the single-source problem in $O(m+n \log \log n)$ time. Both these results are theoretical improvements over Dijkstra's algorithm, which was the previous best for real weighted undirected graphs. Our algorithm takes the hierarchy-based approach invented by Thorup.
\end{abstract}

Key words. single-source shortest paths, all-pairs shortest paths, undirected graphs, Dijkstra's algorithm

AMS subject classifications. 05C12, 05C $85,68 \mathrm{R} 10$

DOI. $10.1137 /$ S0097539702419650

1. Introduction. The problem of computing shortest paths is indisputably one of the most well-studied problems in computer science. It is thoroughly surprising that in the setting of real-weighted graphs, many basic shortest path problems have seen little or no progress since the early work by Dijkstra, Bellman and Ford, Floyd and Warshall, and others [CLRS01]. For instance, no algorithm for computing singlesource shortest paths (SSSPs) in arbitrarily weighted graphs has yet to improve the Bellman-Ford $O(m n)$ time bound, where $m$ and $n$ are the number of edges and vertices, respectively. The fastest uniform all-pairs shortest path (APSP) algorithm for dense graphs [Z04, F76] requires time $O\left(n^{3} \sqrt{\log \log n} / \log n\right)$, which is just a slight improvement over the $O\left(n^{3}\right)$ bound of the Floyd-Warshall algorithm. Similarly, Dijkstra's $O(m+n \log n)$ time algorithm [Dij59, FT87] remains the best for computing SSSPs on nonnegatively weighted graphs, and until the recent algorithms of Pettie [Pet04, Pet02b, Pet03], Dijkstra's algorithm was also the best for computing APSPs on sparse graphs [Dij59, J77, FT87].

In order to improve these bounds most shortest path algorithms depend on a restricted type of input. There are algorithms for geometric inputs (see Mitchell's survey [Mit00]), planar graphs [F91, HKRS97, FR01], and graphs with randomly chosen edge weights [Spi73, FG85, MT87, KKP93, KS98, M01, G01, Hag04]. In recent years there

* Received by the editors December 13, 2002; accepted for publication (in revised form) October 15, 2004; published electronically July 26, 2005. This work was supported by Texas Advanced Research Program grant 003658-0029-1999 and NSF grant CCR-9988160. A preliminary version of this paper, titled Computing shortest paths with comparisons and additions, was presented at the 13th Annual ACM-SIAM Symposium on Discrete Algorithms, 2002, San Francisco, CA.

http://www.siam.org/journals/sicomp/34-6/41965.html

${ }^{\dagger}$ Max Planck Institut für Informatik, Stuhlsatzenhausweg 85, 66123 Saarbrücken, Germany (pettie@mpi-sb.mpg.de). This author's work was also supported by an Alexander von Humboldt Postdoctoral Fellowship and by an MCD Graduate Fellowship.

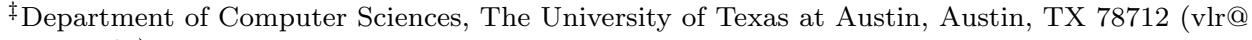
cs.utexas.edu). 
has also been a focus on computing approximate shortest paths - see Zwick's recent survey [Z01]. One common assumption is that the graph is integer-weighted, though structurally unrestricted, and that the machine model is able to manipulate the integer representation of weights. Shortest path algorithms based on scaling [G85b, GT89, G95] and fast matrix multiplication [Sei95, GM97, AGM97, Tak98, SZ99, Z02] have running times that depend on the magnitude of the integer edge weights, and therefore yield improved algorithms only for sufficiently small edge weights. In the case of the matrix multiplication-based algorithms the critical threshold is rather low: even edge weights sublinear in $n$ can be too large. Dijkstra's algorithm can be sped up in the integer-weight model by using an integer priority queue. ${ }^{1}$ The best bounds on Dijkstra's algorithm to date are $O(m \sqrt{\log \log n})$ (expected) [HT02] and $O(m+n \log \log n)$ [Tho03]. Both of these algorithms use multiplication, a non- $A C^{0}$ operation; see [Tho03] for bounds in the $A C^{0}$ model. Thorup [Tho99] considered the restricted case of integer-weighted undirected graphs and showed that on an $A C^{0}$ random access machine (RAM), shortest paths could be computed in linear time. Thorup invented what we call in this paper the hierarchy-based approach to shortest paths.

The techniques developed for integer-weighted graphs (scaling, matrix multiplication, integer sorting, and Thorup's hierarchy-based approach) seem to depend crucially on the graph being integer-weighted. This state of affairs is not unique to the shortest path problem. In the weighted matching [G85b, GT89, GT91] and maximum flow problems [GR98], for instance, the best algorithms for real- and integerweighted graphs have running times differing by a polynomial factor. For the shortest path problem on positively weighted graphs the integer/real gap is only logarithmic. It is of great interest whether an integer-based approach is inherently so, or whether it can yield a faster algorithm for general, real-weighted inputs. In this paper we generalize Thorup's hierarchy-based approach to the comparison-addition model (see section 2.1) and, as a corollary, to real-weighted input graphs. For the undirected APSP problem we nearly eliminate the existing integer/real gap, reducing it from $\log n$ to $\log \alpha(m, n)$, where $\alpha$ is the incomprehensibly slowly growing inverseAckermann function. Before stating our results in detail, we first give an overview of the hierarchy-based approach and discuss the recent hierarchy-based shortest path algorithms [Tho99, Hag00, Pet04, Pet02b].

Hierarchy-based algorithms should be thought of as preprocessing schemes for answering SSSP queries in nonnegatively weighted graphs. The idea is to compute a small non-source-specific structure that encodes useful information about all the shortest paths in the graph. We measure the running time of a hierarchy-based algorithm with two quantities: $\mathcal{P}$, the worst case preprocessing cost on the given graph, and $\mathcal{M}$, the marginal cost of one SSSP computation after preprocessing. Therefore, solving the $s$-sources shortest path problem requires $s \cdot \mathcal{M}+\mathcal{P}$ time. If $s=n$, that is, if we are solving APSP, then for all known hierarchy algorithms the $\mathcal{P}$ term becomes negligible. However, $\mathcal{P}$ may be dominant (in either the asymptotic or real-world sense) for smaller values of $s$. In Thorup's original algorithm [Tho99], $\mathcal{P}$ and $\mathcal{M}$ are both $O(m)$; recall that his algorithm works on integer-weighted undirected graphs. Hagerup [Hag00] adapted Thorup's algorithm to integer-weighted directed graphs, incurring a slight loss of efficiency in the process. In [Hag00], $\mathcal{P}=O(\min \{m \log \log C, m \log n\}),{ }^{2}$

\footnotetext{
${ }^{1}$ It can also be sped up using an integer sorting algorithm in conjunction with Thorup's reduction [Tho00] from priority queues to sorting.

${ }^{2}$ Hagerup actually proved $\mathcal{P}=O(\min \{m \log \log C, m n\})$; see [Pet04] for the $O(m \log n)$ bound.
} 
where $C$ is the maximum edge weight and $\mathcal{M}=O(m+n \log \log n)$. After the initial publication of our results [PR02a], Pettie [Pet04, Pet02b] gave an adaptation of the hierarchy-based approach to real-weighted directed graphs. The main result of [Pet04] is an APSP algorithm running in time $O\left(m n+n^{2} \log \log n\right)$, which improved upon the $O\left(m n+n^{2} \log n\right)$ bound derived from multiple runs of Dijkstra's algorithm [Dij59, J77, FT87]. The result of [Pet04] is stated in terms of the APSP problem because its preprocessing cost $\mathcal{P}$ is $O(m n)$, making it efficient only if $s$ is very close to $n$. In [Pet02b] (see also [Pet03]) the nonuniform complexity of APSP is considered; the main result of [Pet02b] is an algorithm performing $O(m n \log \alpha(m, n))$ comparison and addition operations. This bound is essentially optimal when $m=O(n)$ due to the trivial $\Omega\left(n^{2}\right)$ lower bound on APSP.

In this paper we give new bounds on computing undirected shortest paths in real-weighted graphs. For our algorithm, the preprocessing cost $\mathcal{P}$ is $O(\operatorname{MST}(m, n)+$ $\min \{n \log n, n \log \log r\})$, where $\operatorname{MST}(m, n)$ is the complexity of the minimum spanning tree problem and $r$ is the ratio of the maximum-to-minimum edge weight. This bound on $\mathcal{P}$ is never worse than $O(m+n \log n)$, though if $r$ is not excessively large, say less than $n^{(\log n)^{O(1)}}, \mathcal{P}$ is $O(m+n \log \log n)$. We show that the marginal cost $\mathcal{M}$ of our algorithm is asymptotically equivalent to $\operatorname{SPLIT-Findmin}(m, n)$, which is the decision-tree complexity of a certain data structuring problem of the same name. It was known that $\operatorname{SPLIT-FINDMIN}(m, n)=O(m \alpha(m, n))$ [G85a]; we improve this bound to $O(m \log \alpha(m, n))$. Therefore, the marginal cost of our algorithm is essentially (but perhaps not precisely) linear. Theorem 1.1 gives our general result, and Corollaries 1.2 and 1.3 relate it to the canonical APSP and SSSP problems, respectively.

TheOREM 1.1. Let $\mathcal{P}=\operatorname{MST}(m, n)+\min \{n \log n, n \log \log r\}$, where $m$ and $n$ are the number of edges and vertices in a given undirected graph, $r$ bounds the ratio of any two edge lengths, and $\operatorname{MST}(m, n)$ is the cost of computing the graph's minimum spanning tree. In $O(\mathcal{P})$ time an $O(n)$-space structure can be built that allows the computation of SSSPs in $O(\operatorname{SPLIT-FIndmin}(m, n))$ time, where $\operatorname{Split}-\mathrm{Findmin}(m, n)=$ $O(m \log \alpha(m, n))$ represents the decision-tree complexity of the split-findmin problem and $\alpha$ is the inverse-Ackermann function.

CoROLLARY 1.2. The undirected APSP problem can be solved on a real-weighted graph in $O(n \cdot \operatorname{SPLIT-FINDMin}(m, n))=O(m n \log \alpha(m, n))$ time.

COROLlarY 1.3. The undirected SSSP problem can be solved on a real-weighted graph in $O(\operatorname{SPLIT-FINDMIN}(m, n)+\operatorname{MST}(m, n)+\min \{n \log n, n \log \log r\})=O(m \alpha(m, n)+$ $\min \{n \log n, n \log \log r\})$ time.

The running time of our SSSP algorithm (Corollary 1.3) is rather unusual. It consists of three terms, where the first two are unknown (but bounded by $O(m \alpha(m, n)))$ and the third depends on a nonstandard parameter: the maximum ratio of any two edge lengths. ${ }^{3}$ A natural question is whether our SSSP algorithm can be substantially improved. In section 6 we formally define the class of "hierarchy-based" SSSP algorithms and show that any comparison-based undirected SSSP algorithm in this class must take time $\Omega(m+\min \{n \log n, n \log \log r\})$. This implies that our SSSP algorithm is optimal for this class, up to an inverse-Ackermann factor, and that no hierarchybased SSSP algorithm can improve on Dijkstra's algorithm, for $r$ unbounded.

Pettie, Ramachandran, and Sridhar [PRS02] implemented a simplified version of our algorithm. The observed marginal cost of the [PRS02] implementation is nearly linear, which is in line with our asymptotic analysis. Although it is a little slower

\footnotetext{
${ }^{3}$ Dinic's implementation [Din78, Din03] of Dijkstra's algorithm also depends on $r$, in both time and space consumption.
} 
than Dijkstra's algorithm in solving SSSP, it is faster in solving the $s$-sources shortest path problem, in some cases for $s$ as small as 3. In many practical situations it is the $s$-sources problem, not SSSP, that needs to be solved. For instance, if the graph represents a physical network, such as a network of roads or computers, it is unlikely to change very often. Therefore, in these situations a nearly linear preprocessing cost is a small price to pay for more efficient shortest path computations.

1.1. An overview. In section 2 we define the SSSP and APSP problems and review the comparison-addition model and Dijkstra's algorithm [Dij59]. In section 3 we generalize the hierarchy approach to real-weighted graphs and give a simple proof of its correctness. In section 4 we propose two implementations of the general hierarchy-based algorithm, one for proving the asymptotic bounds of Theorem 1.1 and one that is simpler and uses more standard data structures. The running times of our implementations depend heavily on having a well-balanced hierarchy. In section 5 we give an efficient method for constructing balanced hierarchies; it is based on a hierarchical clustering of the graph's minimum spanning tree. In section 6 we prove a lower bound on the class of hierarchy-based undirected SSSP algorithms. In section 7 we discuss avenues for further research.

2. Preliminaries. The input is a weighted, undirected graph $G=(V, E, \ell)$, where $V=V(G)$ and $E=E(G)$ are the sets of $n$ vertices and $m$ edges, respectively, and $\ell: E \rightarrow \mathbb{R}$ assigns a real length to each edge. The distance from vertex $u$ to vertex $v$, denoted $d(u, v)$, is the length of the minimum length path from $u$ to $v$, or $\infty$ if there is no such path from $u$ to $v$, or $-\infty$ if there is no such path of minimum length. The APSP problem is to compute $d(u, v)$, for $(u, v) \in V \times V$, and the SSSP problem is to compute $d(u, v)$ for some specified source $u$ and all $v \in V$.

If, in an undirected graph, some connected component contains an edge of negative length, say $e$, then the distance between two vertices $u$ and $v$ in that component is $-\infty$ : one can always construct a path of arbitrarily small length by concatenating a path from $u$ to $e$, followed by the repetition of $e$ a sufficient number of times, followed by a path from $e$ to $v$. Without loss of generality we will assume that $\ell: E \rightarrow \mathbb{R}^{+}$assigns only positive edge lengths. A slightly restricted problem (which forbids the types of paths described above) is the shortest simple path problem. This problem is NP-hard as it generalizes the Hamiltonian path problem. However, Edmonds showed that when there is no negative weight simple cycle, the problem is solvable in polynomial time by a reduction to weighted matching - see [AMO93, p. 496] and [G85a].

2.1. The comparison-addition model. We use the term comparison-addition model to mean any uniform model in which real numbers are subject to only comparison and addition operations. The term comparison-addition complexity refers to the number of comparison and addition operations, ignoring other computational costs. In the comparison-addition model we leave unspecified the machine model used for all data structuring tasks. Our results as stated hold when that machine model is a RAM. If instead we assume a pointer machine [Tar79], our algorithms slow down by at most an inverse-Ackermann factor. ${ }^{4}$

The comparison-addition model has some aesthetic appeal because it is the simplest model appropriate to computing shortest paths and many other network opti-

\footnotetext{
${ }^{4}$ The only structure we use whose complexity changes between the RAM and pointer machine models is the split-findmin structure. On a pointer machine there are matching upper and lower bounds of $\Theta(m \alpha)$ [G85a, LaP96], whereas on the RAM the complexity is somewhere between $\Omega(m)$ and $O(m \log \alpha)$-see Appendix B.
} 
mization problems. A common belief is that simplicity is necessarily gained at the price of practicality; however, this is not true. In the setting of an algorithms library, such as LEDA [MN00], it is important - and practical - that data types be fully separated from algorithms and that the interface between the two be as generic as possible. There is always room for fast algorithms specialized to integers or floats. However, even under these assumptions, the gains in speed can be surprisingly minor; see [PRS02] for one example.

2.1.1. Techniques. In our algorithm we sometimes use subtraction on real numbers, an operation that is not directly available in the comparison-addition model. Lemma 2.1, given below, shows that simulating subtraction incurs at most a constant factor loss in efficiency.

Lemma 2.1. $C$ comparisons and $A$ additions and subtractions can be simulated in the comparison-addition model with $C$ comparisons and $2(A+C)$ additions.

Proof. We represent each real $x_{i}=a_{i}-b_{i}$ as two reals $a_{i}, b_{i}$. An addition $x_{i}+x_{j}=$ $\left(a_{i}+a_{j}\right)-\left(b_{i}+b_{j}\right)$ or a subtraction $x_{i}-x_{j}=\left(a_{i}+b_{j}\right)-\left(b_{i}+a_{j}\right)$ can be simulated with two actual additions. A comparison $x_{i}: x_{j}$ is equivalent to the comparison $a_{i}+b_{j}: a_{j}+b_{i}$, which involves two actual additions and a comparison.

At a key point in our algorithm we need to approximate the ratio of two numbers. Division is clearly not available for real numbers in the comparison-addition model, and with a little thought one can see that it cannot be simulated exactly. Lemma 2.2, given below, bounds the time to find certain approximate ratios in the comparisonaddition model, which will be sufficient for our purposes.

LEMma 2.2. Let $p_{1}, \ldots, p_{k}$ be real numbers, where $p_{1}$ and $p_{k}$ are the smallest and largest, respectively. We can find the set of integers $\left\{q_{i}\right\}$ such that $2^{q_{i}} \leq \frac{p_{i}}{p_{1}}<2^{q_{i}+1}$ in $\Theta\left(\log \frac{p_{k}}{p_{1}}+k \log \log \frac{p_{k}}{p_{1}}\right)$ time.

Proof. We generate the set $\mathcal{L}=\left\{p_{1}, 2 \cdot p_{1}, 4 \cdot p_{1}, \ldots, 2^{\left\lceil\log \frac{p_{k}}{p_{1}}\right\rceil} \cdot p_{1}\right\}$ with $\log \frac{p_{k}}{p_{1}}$ additions; then for each $p_{i}$ we find $q_{i}$ in $\log |\mathcal{L}|=O\left(\log \log \frac{p_{k}}{p_{1}}\right)$ time with a binary search over $\mathcal{L}$.

In our algorithm the $\left\{p_{i}\right\}$ correspond to certain edge lengths, and $k=\Theta(n)$. Our need to approximate ratios, as in Lemma 2.2, is the source of the peculiar $n \log \log r$ term in the running time of Theorem 1.1. We note here that the bound stated in Lemma 2.2 is pessimistic in the following sense. If we randomly select the $\left\{p_{i}\right\}$ from a uniform distribution (or other natural distribution), then the time to find approximate ratios can be reduced to $O(k)$ (with high probability) using a linear search rather than a binary search.

2.1.2. Lower bounds. There are many lower bounds for shortest path problems in the comparison-addition model, though none are truly startling. Spira and Pan [SP75] showed that even if additions are free, $\Omega\left(n^{2}\right)$ comparisons are necessary to solve SSSP on the complete graph. Karger, Koller, and Phillips [KKP93] proved that directed APSP requires $\Omega(m n)$ comparisons if each summation corresponds to a path in the graph. ${ }^{5}$ Kerr [K70] showed that any oblivious APSP algorithm performs $\Omega\left(n^{3}\right)$ comparisons, and Kolliopoulos and Stein [KS98] proved that any fixed sequence of edge relaxations solving SSSP must have length $\Omega(m n)$. By "fixed sequence" they mean one that depends only on $m$ and $n$ but not on the graph structure. Ahuja et al. [AMOT90] observed that any implementation of Dijkstra's algorithm requires $\Omega(m+n \log n)$ comparison and addition operations. Pettie [Pet04] gave an $\Omega(m+$

\footnotetext{
${ }^{5}$ However, it is not true that all shortest path algorithms satisfy this condition. For example, our algorithm does not, and neither do [F76, Tak92, Han04, Z04, Pet04, Pet02b].
} 
$\min \{n \log r, n \log n\})$ lower bound on computing directed SSSP with a "hierarchytype" algorithm, where $r$ bounds the ratio of any two edge lengths. In section 6 we prove a lower bound of $\Omega(m+\min \{n \log \log r, n \log n\})$ on hierarchy-type algorithms for undirected SSSP. These last two lower bounds are essentially tight for hierarchytype algorithms, on directed and undirected graphs, respectively.

Graham, Yao, and Yao [GYY80] proved that the information-theoretic argument cannot prove a nontrivial $\omega\left(n^{2}\right)$ lower bound on the comparison-complexity of APSP, where additions are granted for free. It is also simple to see that there can be no nontrivial information-theoretic lower bound on SSSP.

2.2. Dijkstra's algorithm. Our algorithm, like [Tho99, Hag00], is best understood as circumventing the limitations of Dijkstra's algorithm. We give a brief description of Dijkstra's algorithm in order to illustrate its complexity and introduce some vocabulary.

For a vertex set $S \subseteq V(G)$, let $d_{S}(u, v)$ denote the distance from $u$ to $v$ in the subgraph induced by $S \cup\{v\}$. Dijkstra's algorithm maintains a tentative distance function $D(v)$ and a set of visited vertices $S$ satisfying Invariant 2.1. Henceforth, $s$ denotes the source vertex.

INVARIANT 2.1. Let $s$ be the source vertex and $v$ be an arbitrary vertex:

$$
D(v)= \begin{cases}d(s, v) & \text { if } v \in S, \\ d_{S}(s, v) & \text { if } v \notin S .\end{cases}
$$

Choosing an initial assignment of $S=\emptyset, D(s)=0$, and $D(v)=\infty$ for $v \neq s$ clearly satisfies the invariant. Dijkstra's algorithm consists of repeating the following step $n$ times: choose a vertex $v \in V(G) \backslash S$ such that $D(v)$ is minimized, set $S:=$ $S \cup\{v\}$, and finally, update tentative distances to restore Invariant 2.1. This last part involves relaxing each edge $(v, w)$ by setting $D(w)=\min \{D(w), D(v)+\ell(v, w)\}$. Invariant 2.1 and the positive-weight assumption imply $D(v)=d(s, v)$ when $v$ is selected. It is also simple to prove that relaxing outgoing edges of $v$ restores Invariant 2.1 .

The problem with Dijkstra's algorithm is that vertices are selected in increasing distance from the source, a task that is at least as hard as sorting $n$ numbers. Maintaining Invariant 2.1, however, does not demand such a particular ordering. In fact, it can be seen that selecting any vertex $v \notin S$ for which $D(v)=d(s, v)$ will maintain Invariant 2.1. All hierarchy-type algorithms [Tho99, Hag00, Pet04, Pet02b] maintain Invariant 2.1 by generating a weaker certificate for $D(v)=d(s, v)$ than " $D(v)$ is minimal." Any such certificate must show that for all $u \notin S, D(u)+d(u, v) \geq D(v)$. For example, Dijkstra's algorithm presumes there are no negative length edges, hence $d(u, v) \geq 0$, and by choice of $v$ ensures $D(u) \geq D(v)$. This is clearly a sufficient certificate. In Dinic's version [Din78] of Dijkstra's algorithm the lower bound $d(u, v) \geq \ell_{\min }$ is used, where $\ell_{\min }$ is the minimum edge length. Thus Dinic is free to visit any $v \notin S$ for which $\left\lfloor D(v) / \ell_{\min }\right\rfloor$ is minimal. All hierarchy-type algorithms [Tho99, Hag00, Pet04, Pet02b], ours included, precompute a much stronger lower bound on $d(u, v)$ than $d(u, v) \geq \ell_{\min }$.

3. The hierarchy approach and its correctness. In this section we generalize the hierarchy-based approach of [Tho99] to real-weighted graphs. Because the algorithm follows directly from its proof of correctness, we will actually give a kind of correctness proof first.

Below, $X \subseteq V(G)$ denotes any set of vertices, and $s$ always denotes the source vertex. Let $I$ be a real interval. The notation $X^{I}$ refers to the subset of $X$ whose 
distance from the source lies in the interval $I$, i.e.,

$$
X^{I}=\{v \in X: d(s, v) \in I\} .
$$

Definition 3.1. A vertex set $X$ is $(S,[a, b))$-SAFE if (i) $X^{[0, a)} \subseteq S$,

(ii) for $v \in X^{[a, b)}, d_{S \cup X}(s, v)=d(s, v)$.

In other words, if a subgraph is $(S, I)$-SAFE, we can determine the distances that lie in interval $I$ without looking at parts of the graph outside the subgraph and $S$. Clearly, finding safe subgraphs has the potential to let us compute distances cheaply.

Definition 3.2. A set $\left\{X_{i}\right\}_{i}$ is a t-partition of $X$ if the $\left\{X_{i}\right\}_{i}$ partition $X$ and for every edge $(u, v)$ with $u \in X_{i}, v \in X_{j}$, and $i \neq j$, we have $\ell(u, v) \geq t$.

Note that a $t$-partition need not be maximal; that is, if $\left\{X_{1}, X_{2}, \ldots, X_{k}\right\}$ is a $t$-partition, then $\left\{X_{1} \cup X_{2}, X_{3}, \ldots, X_{k}\right\}$ is as well.

Lemma 3.3. Suppose that $X$ is $(S,[a, b))$-SAFE. Let $\left\{X_{i}\right\}_{i}$ be a t-partition of $X$ and let $S^{\prime}$ be such that $S \cup X^{[a, \min \{a+t, b\})} \subseteq S^{\prime}$. Then

(i) for $X_{i}$ in the t-partition, $X_{i}$ is $(S,[a, \min \{a+t, b\}))$-SAFE;

(ii) $X$ is $\left(S^{\prime},[\min \{a+t, b\}, b)\right)$-SAFE.

Proof. We prove part (i) first. Let $v \in X_{i}^{[a, \min \{a+t, b\})}$ and suppose that the lemma is false, that $d(s, v) \neq d_{S \cup X_{i}}(s, v)$. From the assumed safeness of $X$ we know that $d(s, v)=d_{S \cup X}(s, v)$. This means that the shortest path to $v$ must pass through $X \backslash\left(X_{i} \cup S\right)$. Let $w$ be the last vertex in $X \backslash\left(X_{i} \cup S\right)$ on the shortest $s-v$ path. By Definition 3.2, the edge from $w$ to $X_{i}$ has length $\geq t$. Since $d(s, v)<\min \{a+t, b\}$, $d(s, w)<\min \{a+t, b\}-t \leq a$. Since, by Definition 3.1(i), $X^{[0, a)} \in S$, it must be that $w \in S$, contradicting our selection of $w$ from $X \backslash\left(X_{i} \cup S\right)$. Part (ii) claims that $X$ is $\left(S^{\prime},[\min \{a+t, b\}, b)\right)$-SAFE. Consider first Definition 3.1(i) regarding safeness. By the assumption that $X$ is $(S,[a, b))$-SAFE we have $X^{[0, a)} \subseteq S$, and by definition of $S^{\prime}$ we have $S \cup X^{[a, \min \{a+t, b\})} \subseteq S^{\prime}$; therefore $X^{[0, \min \{a+t, b\})} \subseteq S^{\prime}$, satisfying Definition 3.1(i). By the assumption that $X$ is $(S,[a, b))$-SAFE we have that for $v \in X^{[a, b)}$, $d_{S \cup X}(s, v)=d(s, v)$; this implies the weaker statement that for $v \in X^{[\min \{a+t, b\}, b)}$, $d_{S^{\prime} \cup X}(s, v)=d_{S \cup X}(s, v)=d(s, v)$.

As Thorup noted [Tho99], Lemma 3.3 alone leads to a simple recursive procedure for computing SSSP; however, it makes no guarantee as to efficiency. The input to the procedure is an $(S, I)$-SAFE subgraph $X$; its only task is to compute the set $X^{I}$, which it performs with recursive calls (corresponding to Lemma 3.3(i) and (ii)) or directly if $X$ consists of a single vertex. There are essentially three major obstacles to making this general algorithm efficient: bounding the number of recursive calls, bounding the time to decide what those recursive calls are, and computing good $t$ partitions. Thorup gave a simple way to choose the $t$-partitions in integer-weighted graphs so that the number of recursive calls is $O(n)$. However, if adapted directly to the comparison-addition model, the time to decide which calls to make becomes $\Omega(n \log n)$; it amounts to the problem of implementing a general priority queue. We reduce the overhead for deciding which recursive calls to make to linear by using a "well balanced" hierarchy and a specialized priority queue for exploiting this kind of balance. Our techniques rely heavily on the graph being undirected and do not seem to generalize to directed graphs in any way.

As in other hierarchy-type algorithms, we generalize the distance and tentative distance notation from Dijkstra's algorithm to include not just single vertices but sets of vertices. If $X$ is a set of vertices (or associated with a set of vertices), then

$$
D(X) \stackrel{\text { def }}{=} \min _{v \in X} D(v) \quad \text { and } \quad d(u, X) \stackrel{\text { def }}{=} \min _{v \in X} d(u, v) .
$$


The procedure Generalized-Visit, given below, takes a vertex set $X$ that is $(S, I)$-SAFE and computes the distances to all vertices in $X^{I}$, placing these vertices in the set $S$ as their distances become known. We maintain Invariant 2.1 at all times. By Definition 3.1 we can compute the set $X^{I}$ without looking at parts of the graph outside of $S \cup X$. If $X=\{v\}$ happens to contain a single vertex, we can compute $X^{I}$ directly: if $D(v) \in I$, then $X^{I}=\{v\}$; otherwise it is $\emptyset$. For the general case, Lemma 3.3 says that we can compute $X^{I}$ by first finding a $t$-partition $\chi$ of $X$, then computing $X^{I}$ in phases. Let $I=I_{1} \cup I_{2} \cup \cdots \cup I_{k}$, where each subinterval is disjoint from the others and has width $t$, except perhaps $I_{k}$, which may be a leftover interval of width less than $t$. Let $S_{i}=S \cup X^{I_{1}} \cup \cdots \cup X^{I_{i}}$ and let $S_{0}=S$. By the assumption that $X$ is $(S, I)$-SAFE and Lemma 3.3 , each set in $\chi$ is $\left(S_{i}, I_{i+1}\right)$-SAFE. Therefore, we can compute $S_{1}, S_{2}, \ldots, S_{k}=S \cup X^{I}$ with a series of recursive calls as follows. Assume that the current set of visited vertices is $S_{i}$. We determine $X^{I_{i+1}}=\bigcup_{Y \in \chi} Y^{I_{i+1}}$ with recursive calls of the form GenERALized-Visit $\left(Y, I_{i+1}\right)$, for every $Y \in \chi$ such that $Y^{I_{i+1}} \neq \emptyset$.

To start things off, we initialize the set $S$ to be empty, set the $D$-values (tentative distances) according to Invariant 2.1, and call Generalized-Visit $(V(G),[0, \infty))$. By the definition of safeness, $V(G)$ is clearly $(\emptyset,[0, \infty))$-SAFE. If GeneraLIZED-Visit works according to specification, when it completes $S=V(G)$ and Invariant 2.1 is satisfied, implying that $D(v)=d(s, v)$ for all vertices $v \in V(G)$.

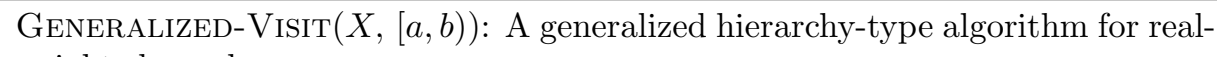
weighted graphs.

Input guarantee: $X$ is $(S,[a, b))$-SAFE and Invariant 2.1 is satisfied.

Output guarantee: Invariant 2.1 is satisfied and $S_{\text {post }}=S_{\text {pre }} \cup X^{[a, b)}$, where $S_{\text {pre }}$ and $S_{\text {post }}$ are the set $S$ before and after the call.

1. If $X$ contains one vertex, $X=\{v\}$, and $D(v) \in[a, b)$, then $D(v)=$ $d_{S}(s, v)=d(s, v)$, where the first equality is by Invariant 2.1 and the second by the assumption that $X$ is $(S,[a, b))$-SAFE. Let $S:=S \cup\{v\}$. Relax all edges incident on $v$, restoring Invariant 2.1, and return.

2. Let $a^{\prime}:=a$

While $a^{\prime}<b$ and $X \nsubseteq \subseteq$

Let $t>0$ be any positive real

Let $\chi=\left\{X_{1}, X_{2}, \ldots, X_{k}\right\}$ be an arbitrary $t$-partition of $X$

Let $\chi^{\prime}=\left\{X_{i} \in \chi: D\left(X_{i}\right)<\min \left\{a^{\prime}+t, b\right\}\right.$ and $\left.X_{i} \not \subseteq S\right\}$

For each $X_{i} \in \chi^{\prime}, \operatorname{Generalized-\operatorname {Visit}}\left(X_{i},\left[a^{\prime}, \min \left\{a^{\prime}+t, b\right\}\right)\right)$

$a^{\prime}:=\min \left\{a^{\prime}+t, b\right\}$

LEMMA 3.4. If the input guarantees of GENERALIZED-VISIT are met, then after a call to Generalized-Visit $(X, I)$, Invariant 2.1 remains satisfied and $X^{I}$ is a subset of the visited vertices $S$.

Proof (sketch). The base case, when $X$ is a single vertex, is simple to handle. Turning to the general case, we prove that each time the while statement is examined in step 2, $X$ is $\left(S,\left[a^{\prime}, b\right)\right)$-SAFE for the current value of $S$ and $a^{\prime}$; in what follows we will treat $S$ as a variable, not a specific vertex set. The first time through the whileloop in step 2, it follows from the input guarantee to Generalized-Visit that $X$ is $\left(S,\left[a^{\prime}, b\right)\right)$-SAFE. Similarly, the input guarantee for all recursive calls holds by Lemma 3.3. However, to show that $X$ is $\left(S,\left[a^{\prime}, b\right)\right)$-SAFE at the assignment $a^{\prime}:=\min \left\{a^{\prime}+t, b\right\}$, by Definition 3.1 we must show $X^{\left[0, \min \left\{a^{\prime}+t, b\right\}\right)} \subseteq S$. We assume inductively that the output guarantee of any recursive call to GENERALIZED-VISIT is fulfilled; that is, 
upon the completion of Generalized-Visit $\left(X_{i},\left[a^{\prime}, \min \left\{a^{\prime}+t, b\right\}\right)\right), S$ includes the set $X_{i}^{\left[a^{\prime}, \min \left\{a^{\prime}+t, b\right\}\right)}$. Each time through the while-loop in step 2 GENERALIZED-VISIT makes recursive calls to all $Y \in \chi^{\prime}$. To complete the proof we must show that for $Y \in \chi \backslash \chi^{\prime}, Y^{\left[a^{\prime}, \min \left\{a^{\prime}+t, b\right\}\right)} \backslash S=\emptyset$. If $Y \in \chi \backslash \chi^{\prime}$, it was because $D(Y) \geq \min \left\{a^{\prime}+t, b\right\}$ or because $Y \subseteq S$, both of which clearly imply $Y^{\left[a^{\prime}, \min \left\{a^{\prime}+t, b\right\}\right)} \backslash S=\emptyset$. The output guarantee for GENERALIZED-VISIT is clearly satisfied if step 1 is executed; if step 2 is executed, then when the while-loop finishes, $X$ is either $(S,[b, b))$-SAFE or $X \subseteq S$, both implying $X^{[0, b)} \in S$.

GENERALIZED-Visit can be simplified in a few minor ways. It can be seen that in step 1 we do not need to check whether $D(v) \in[a, b)$; the recursive call would not have taken place were this not the case. In step 2 the final line can be shortened to $a^{\prime}:=a^{\prime}+t$. However, we cannot change all occurrences of $\min \left\{a^{\prime}+t, b\right\}$ to $a^{\prime}+t$ because this is crucial to the procedure's correctness. It is not assumed (nor can it be guaranteed) that $t$ divides $(b-a)$, so the procedure must be prepared to deal with fractional intervals of width less than $t$. In section 4 we show that for a proper hierarchy this fractional interval problem does not arise.

4. Efficient implementations of Generalized-Visit. We propose two implementations of the Generalized-Visit algorithm, called Visit and Visit-B. The time bound claimed in Theorem 1.1 is proved by analyzing VISIT, given later in this section. Although VISIT is asymptotically fast, it seems too impractical for a real-world implementation. In section 4.5 we give the VISIT-B implementation of Generalized-Visit, which uses fewer specialized data structures. The asymptotic running time of VISIT-B is just a little slower than that of VISIT.

VISIT and Visit-B differ from Generalized-Visit in their input/output specification only slightly. Rather than accepting a set of vertices, as GENERALIZED-VISIT does, our implementations (like [Tho99, Hag00, Pet04, Pet02b]) accept a hierarchy node $x$, which represents a set of vertices. Both of our implementations work correctly for any proper hierarchy $\mathcal{H}$, defined below. We prove bounds on their running times as a function of $m, n$, and a certain function of $\mathcal{H}$ (which is different for VIsIT and VISIT-B). In order to compute SSSP in near-linear time the proper hierarchy $\mathcal{H}$ must satisfy certain balance conditions, which are the same for VISIT and VISIT-B. In section 5 we give the requisite properties of a balanced hierarchy and show how to construct a balanced proper hierarchy in $O(\operatorname{MST}(m, n)+\min \{n \log n, n \log \log r\})$ time. Definition 4.1, given next, describes exactly what is meant by hierarchy and proper hierarchy.

Definition 4.1. A hierarchy is a rooted tree whose leaf nodes correspond to graph vertices. If $x$ is a hierarchy node, then $p(x)$ is its parent, $\mathrm{DEG}(x)$ is the number of children of $x, V(x)$ is the set of descendant leaves (or the equivalent graph vertices), and $\operatorname{DIAM}(x)$ is an upper bound on the diameter of $V(x)$ (where the diameter of $V(x)$ is defined to be $\max _{u, v \in V(x)} d(u, v)$ ). Each node $x$ is given a value $\operatorname{NORM}(x)$. A hierarchy is proper if the following hold:

(i) $\operatorname{NORM}(x) \leq \operatorname{NORM}(p(x))$,

(ii) either $\operatorname{NORM}(p(x)) / \operatorname{NORM}(x)$ is an integer or $\operatorname{DIAM}(x)<\operatorname{NORM}(p(x))$,

(iii) $\operatorname{DEG}(x) \neq 1$,

(iv) if $x_{1}, \ldots, x_{\mathrm{DEG}(x)}$ are the children of $x$, then $\left\{V\left(x_{i}\right)\right\}_{i}$ is a $\operatorname{NORM}(x)$-partition of $V(x)$. (Refer to Definition 3.2 for the meaning of "NORM $(x)$-partition.")

Part (iv) of Definition 4.1 is the crucial one for computing shortest paths. Part (iii) guarantees that a proper hierarchy has $O(n)$ nodes. The second part of (ii) is admittedly a little strange. It allows us to replace all occurrences of $\min \{a+t, b\}$ 
in Generalized-Visit with just $a+t$, which greatly simplifies the analysis of our algorithms. Part (i) will be useful when bounding the total number of recursive calls to our algorithms.

4.1. Visit. Consider the Visit procedure given below. We prove that Visit correctly computes SSSPs by demonstrating that it is an implementation of GENERALIZEDVISIT, which was already proved correct.

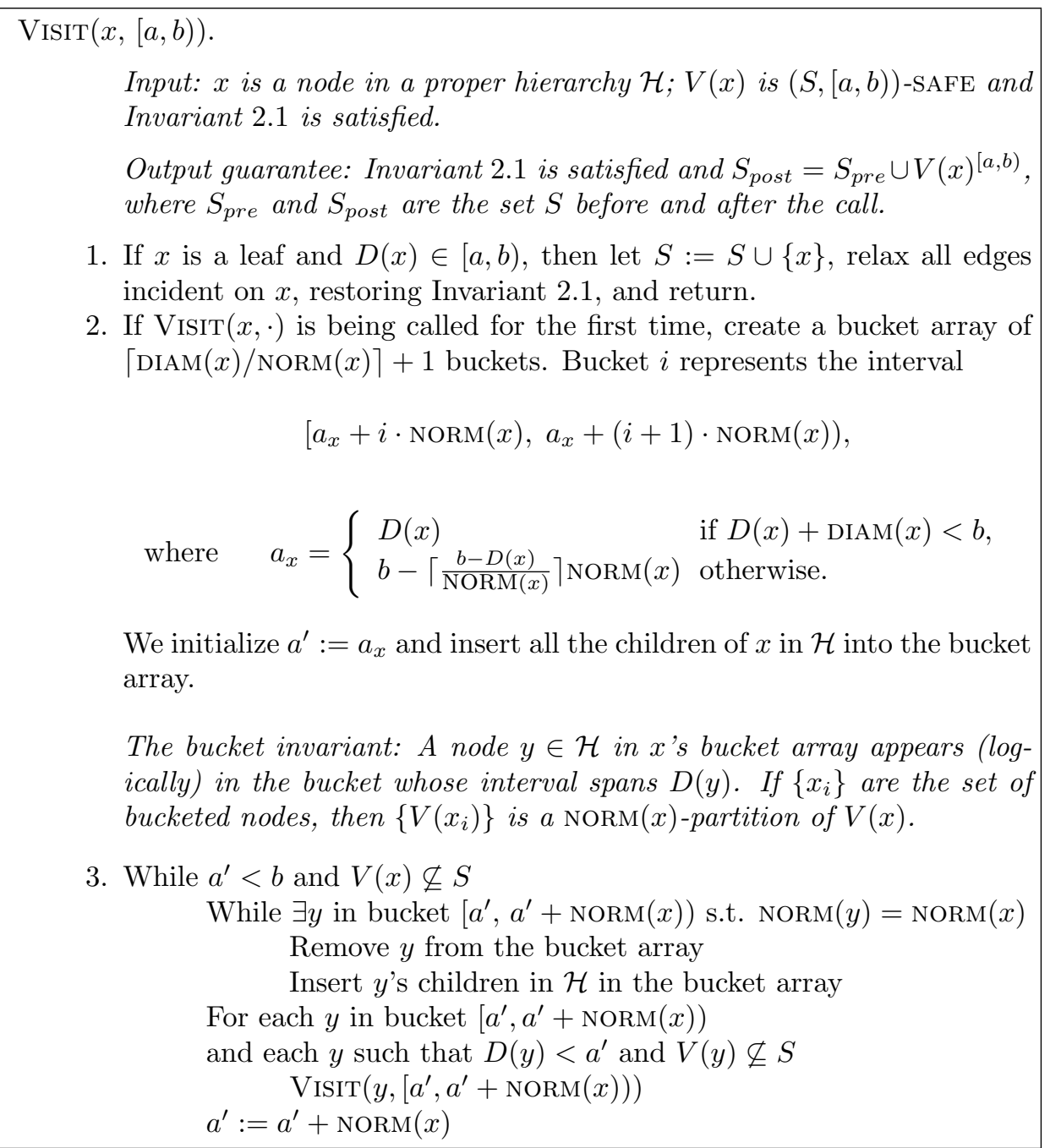

In step 2 of GENERALIZED-VISIT we let $\chi$ be any arbitrary $t$-partition of the subset of vertices given as input. In VIsit the input is a hierarchy node $x$, and the associated vertex set is $V(x)$. We represent the $t$-partition of $V(x)$ (where $t=\operatorname{NORM}(x)$ ) by the set of bucketed $\mathcal{H}$-nodes $\left\{x_{i}\right\}_{i}$ (see step 2), where the sets $\left\{V\left(x_{i}\right)\right\}_{i}$ partition $V(x)$. Clearly the $\left\{x_{i}\right\}_{i}$ are descendants of $x$. The set $\left\{x_{i}\right\}_{i}$ will begin as $x$ 's children, though later on $\left\{x_{i}\right\}_{i}$ may contain a mixture of children of $x$, grandchildren of $x$, and so on.

Consider the inner while-loop in step 3. Assuming inductively that the bucketed $\mathcal{H}$-nodes represent a $\operatorname{NORM}(x)$-partition of $V(x)$, if $y$ is a bucketed node and $\operatorname{NORM}(y)=\operatorname{NORM}(x)$, then replacing $y$ by its children in the bucket array produces a new $\operatorname{NORM}(x)$-partition. This follows from the definitions of $t$-partitions and proper 
Case 1: Fully Aligned

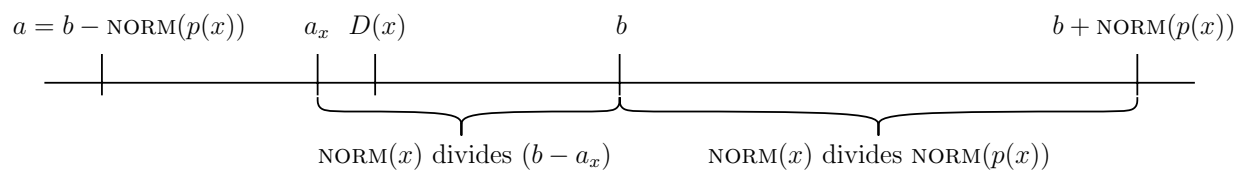

Case 2: Aligned With $b$

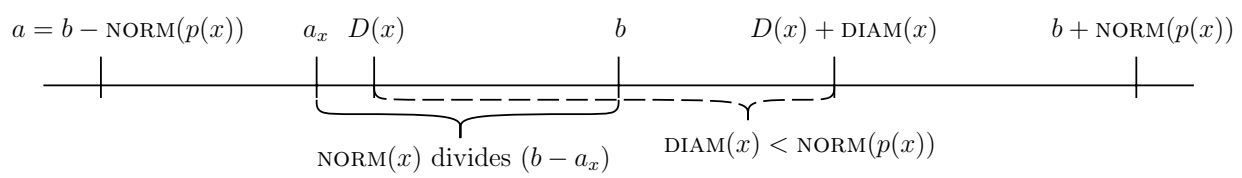

Case 3: Not Aligned At All

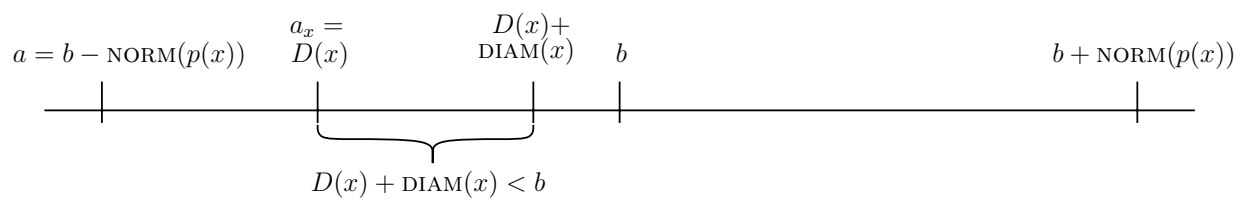

FIG. 1. First observe that when $a_{x}$ is initialized we have $D(x) \geq a_{x} \geq a$, as in the figure. If $a_{x}$ is chosen such that $\operatorname{NORM}(x)$ divides $\left(b-a_{x}\right)$, then by Definition 4.1(ii) either NORM $(x)$ divides $\operatorname{NORM}(p(x))$ (which puts us in Case 1) or $\operatorname{DIAM}(x)<\operatorname{NORM}(p(x))$ (putting us in Case 2); that is, $\operatorname{NORM}(x)$ does not divide $\left(b+\operatorname{NORM}(p(x))-a_{x}\right)$, but it does not matter since we'll never reach $b+\operatorname{NORM}(p(x))$ anyway. If $a_{x}$ is chosen so that $\operatorname{NORM}(x)$ does not divide $\left(b-a_{x}\right)$, then $a_{x}=D(x)$ and $D(x)+\operatorname{DIAM}(x)<b$ (putting us in Case 3 ), meaning we will never reach $b$. Note that by the definition of $\operatorname{DIAM}(x)$ (Definition 4.1) and Invariant 2.1, for any vertex in $u \in V(x)$ we have $d(s, u) \leq d(s, x)+\operatorname{DIAM}(x) \leq D(x)+\operatorname{DIAM}(x)$.

hierarchies (Definitions 3.2 and 4.1). Since the bucketed nodes form a NORM $(x)$ partition, one can easily see that the recursive calls in step 3 of VISIT correspond to the recursive calls in GENERALIZED-VISIT. However, their interval arguments are different. We sketch below why this change does not affect correctness.

In Generalized-Visit the intervals passed to recursive calls are of the form $\left[a^{\prime}, \min \left\{a^{\prime}+t, b\right\}\right)$, whereas in VISIT they are $\left[a^{\prime}, a^{\prime}+t\right)=\left[a^{\prime}, a^{\prime}+\operatorname{NORM}(x)\right)$. We will argue why $a^{\prime}+t=a^{\prime}+\operatorname{NORM}(x)$ is never more than $b$. The main idea is to show that we are always in one of the three cases portrayed in Figure 1.

If $\operatorname{NORM}(x)$ divides $\operatorname{NORM}(p(x))$ and $a_{x}$ is chosen in step 2 so that $t=\operatorname{NORM}(x)$ divides $\left(b-a_{x}\right)$, then we can freely substitute the interval $\left[a^{\prime}, a^{\prime}+t\right)$ for $\left[a^{\prime}, \min \left\{a^{\prime}+\right.\right.$ $t, b\})$ since they will be identical. Note that in our algorithm $(b-a)=\operatorname{NORM}(p(x)){ }^{6}$ The problems arise when $\operatorname{NORM}(x)$ does not divide either $\operatorname{NORM}(p(x))$ or $\left(b-a_{x}\right)$. In order to prove the correctness of VISIT we must show that the input guarantee (regarding SAFE-ness) is satisfied for each recursive call. We consider two cases: when we are in the first recursive call to $\operatorname{Visit}(x, \cdot)$ and any subsequent call. Suppose we are in the first recursive call to $\operatorname{Visit}(x, \cdot)$. By our choice of $a_{x}$ in step 2, either $b=a_{x}+q \cdot \operatorname{NORM}(x)$ for some integer $q$, or $b>D(x)+\operatorname{DIAM}(x)=a_{x}+\operatorname{DIAM}(x)$. If it is the first case, each time the outer while-loop is entered we have $a^{\prime}<b$, which,

\footnotetext{
${ }^{6}$ Strictly speaking, this does not hold for the initial call because in this case, $x=\operatorname{ROOT}(\mathcal{H})$ is the root of the hierarchy $\mathcal{H}$ and there is no such node $p(x)$. The argument goes through just fine if we let $p(\operatorname{ROOT}(\mathcal{H}))$ denote a dummy node such that $\operatorname{NORM}(p(\operatorname{ROOT}(\mathcal{H})))=\infty$.
} 
since $q$ is integral, implies $\min \left\{a^{\prime}+\operatorname{NORM}(x), b\right\}=a^{\prime}+\operatorname{NORM}(x)$. Now consider the second case, where $b>D(x)+\operatorname{DIAM}(x)=a_{x}+\operatorname{DIAM}(x)$, and one of the recursive calls $\operatorname{Visit}\left(y,\left[a^{\prime}, a^{\prime}+\operatorname{NORM}(x)\right)\right)$ made in step 3. By Lemma $3.3, V(y)$ is $\left(S,\left[a^{\prime}, \min \left\{a^{\prime}+\right.\right.\right.$ $\operatorname{NORM}(x), b\}))$-SAFE, and it is actually $\left(S,\left[a^{\prime}, a^{\prime}+\operatorname{NORM}(x)\right)\right)$-SAFE as well because $b>D(x)+\operatorname{DIAM}(x)$, implying $V(y)^{[b, \infty)} \subseteq V(x)^{[b, \infty)}=\emptyset$. (Recall from Definition 4.1 that for any $u \in V(x)$, $\operatorname{DiAM}(x)$ satisfies $d(s, x) \leq d(s, u) \leq d(s, x)+\operatorname{DIAM}(x) \leq$ $D(x)+\operatorname{Diam}(x)$.$) Now consider a recursive call \operatorname{Visit}(x,[a, b))$ that is not the first call to $\operatorname{Visit}(x, \cdot)$. Then by Definition 4.1(ii), either $(b-a)=\operatorname{NORM}(p(x))$ is a multiple of $\operatorname{NORM}(x)$ or $a+\operatorname{DiAM}(x)<b$; these are identical to the two cases treated above.

There are two data structural problems that need to be solved in order to efficiently implement VISIT. First, we need a way to compute the tentative distances of hierarchy nodes, i.e., the $D$-values as defined in (1) in section 3. For this problem we use an improved version of Gabow's split-findmin structure [G85a]. The other problem is efficiently implementing the various bucket arrays, which we solve with a new structure called the bucket-heap. The specifications for these two structures are discussed below, in sections 4.2 and 4.3, respectively. The interested reader can refer to Appendices A and B for details about our implementations of split-findmin and the bucket-heap, and for proofs of their respective complexities.

4.2. The split-findmin structure. The split-findmin structure operates on a collection of disjoint sequences, consisting in total of $n$ elements, each with an associated key. The idea is to maintain the smallest key in each sequence under the following operations.

split $(x)$ : Split the sequence containing $x$ into two sequences: the elements up to and including $x$, and the rest.

decrease-key $(x, \kappa): \quad \operatorname{Set} \operatorname{key}(x)=\min \{\operatorname{key}(x), \kappa\}$.

findmin $(x)$ : Return the element with minimum key in $x$ 's sequence.

Theorem 4.2, given below, establishes some new bounds on the problem that are just slightly better than Gabow's original data structure [G85a]. Refer to Appendix B for a proof. Thorup [Tho99] gave a similar data structure for integer keys in the RAM model that runs in linear time. It relies on the RAM's ability to sort small sets of integers in linear time [FW93].

THEOREM 4.2. The split-findmin problem can be solved on a pointer machine in $O(n+m \alpha)$ time while making only $O(n+m \log \alpha)$ comparisons, where $\alpha=\alpha(m, n)$ is the inverse-Ackermann function. Alternatively, split-findmin can be solved on a RAM in time $\Theta(\operatorname{SPLIT-FIndmin}(m, n))$, where $\operatorname{SPLIT-FIndmin}(m, n)=O(n+m \log \alpha)$ is the decision-tree complexity of the problem.

We use the split-findmin structure to maintain $D$-values as follows. In the beginning there is one sequence consisting of the $n$ leaves of $\mathcal{H}$ in an order consistent with some depth-first search traversal of $\mathcal{H}$. For any leaf $v$ in $\mathcal{H}$ we maintain, by appropriate decrease-key operations, that $\operatorname{key}(v)=D(v)$. During execution of VIsIT we will say an $\mathcal{H}$-node is unresolved if it lies in another node's bucket array but its tentative distance $(D$-value) is not yet finalized. The $D$-value of an $\mathcal{H}$-node becomes finalized, in the sense that it never decreases again, during step 3 of VISIT, either by being removed from some bucket array or passed, for the first time, to a recursive call of Visit. (It follows from Definition 3.1 and Invariant 2.1 that $D(y)=d(s, y)$ at the first recursive call to $y$.) One can verify a couple properties of the unresolved nodes. 
First, each unvisited leaf has exactly one unresolved ancestor. Second, to implement VISIT we need only query the $D$-values of unresolved nodes. Therefore, we maintain that for each unresolved node $y$, there is some sequence in the split-findmin structure corresponding to $V(y)$, the descendants of $y$. Now suppose that a previously unresolved node $y$ is resolved in step 3 of VISIT. The DEG $(y)$ children of $y$ will immediately become unresolved, so to maintain our correspondence between sequences and unresolved nodes, we perform $\operatorname{DEG}(y)-1$ split operations on $y$ 's sequence, so that the resulting subsequences correspond to $y$ 's children.

We remark that the split-findmin structure we use can be simplified slightly because we know in advance where the splits will occur. However, this knowledge does not seem to affect the asymptotic complexity of the problem.

4.3. The bucket-heap. We now turn to the problem of efficiently implementing the bucket array used in Visit. Because of the information-theoretic bottleneck built into the comparison-addition model, we cannot always bucket nodes in constant time: each comparison extracts at most one bit of information, whereas properly bucketing a node in $x$ 's bucket array requires us to extract up to $\log (\operatorname{DIAM}(x) / \operatorname{NORM}(x))$ bits of information. Thorup [Tho99] and Hagerup [Hag00] assume integer edge lengths and the RAM model and therefore do not face this limitation. We now give the specification for the bucket-heap, a structure that supports the bucketing operations of VISIT. This structure logically operates on a sequence of buckets; however, our implementation is really a simulation of the logical structure. Lemma 4.3, proved in Appendix A, bounds the complexity of our implementation of the bucket-heap.

$$
\begin{aligned}
\operatorname{create}(\mu, \delta): & \text { Create a new bucket-heap whose buckets are associated } \\
& \text { with intervals }[\delta, \delta+\mu),[\delta+\mu, \delta+2 \mu),[\delta+2 \mu, \delta+3 \mu), \ldots \\
& \text { An item } x \text { lies in the bucket whose interval spans } \operatorname{key}(x) . \\
& \text { All buckets are initially open. } \\
\operatorname{insert}(x, \kappa): & \text { Insert a new item } x \text { with } \operatorname{key}(x)=\kappa . \\
\text { decrease-key }(x, \kappa): & \text { Set key }(x)=\min \{\operatorname{key}(x), \kappa\} . \text { It is guaranteed that } x \text { is } \\
& \text { not moved to a closed bucket. } \\
\text { enumerate: } & \text { Close the first open bucket and enumerate its contents. }
\end{aligned}
$$

LEMma 4.3. Let $\Delta_{x} \geq 1$ denote the number of buckets between the first open bucket at the time of $x$ 's insertion and the bucket from which $x$ was enumerated. The bucket-heap can be implemented on a pointer machine to run in $O\left(N+\sum_{x} \log \Delta_{x}\right)$ time, where $N$ is the number of operations.

When $\operatorname{Visit}(x, \cdot)$ is called for the first time, we initialize the bucket-heap at $x$ with a call to create $\left(\operatorname{NORM}(x), a_{x}\right)$, followed by a number of insert operations for each of $x$ 's children, where the key of a child is its $D$-value. Here $a_{x}$ is the beginning of the real interval represented by the bucket array, and $\operatorname{NORM}(x)$ the width of each bucket. Every time the $D$-value of a bucketed node decreases, which can easily be detected with the split-findmin structure, we perform a decrease-key on the corresponding item in the bucket-heap. We usually refer to buckets not by their cardinal number but by their associated real interval, e.g., bucket $\left[a_{x}, a_{x}+\operatorname{NORM}(x)\right)$.

4.4. Analysis of Visit. In this section we bound the time required to compute SSSP with VISIT as a function of $m, n$, and the given hierarchy $\mathcal{H}$. We will see later that the dominant term in this running time corresponds to the split-findmin 
structure, whose complexity is no more than $O(m \log \alpha)$ but could turn out to be linear.

Lemma 4.4. Let $\mathcal{H}$ be a proper hierarchy. Computing SSSPs with VISIT on $\mathcal{H}$ takes time $O(\operatorname{split-findmin}(m, n)+\phi(\mathcal{H}))$, where $\operatorname{SPLiT-findmin}(m, n)$ is the complexity of the split-findmin problem and

$$
\phi(\mathcal{H})=\sum_{\substack{x \in \mathcal{H} \\ \text { sorm }(x) \neq \operatorname{NoRm}(p(x))}} \frac{\operatorname{DiAm}(x)}{\operatorname{NORM}(x)}+\sum_{x \in \mathcal{H}} \log \left(\frac{\operatorname{Diam}(p(x))}{\operatorname{NORm}(p(x))}+1\right) .
$$

Proof. The Split-findmin $(m, n)$ term represents the time to relax edges (in step 1 ) and update the relevant $D$-values of $\mathcal{H}$-nodes, as described in section 4.2. Except for the costs associated with updating $D$-values, the overall time of VISIT is linear in the number of recursive calls and the bucketing costs. The two terms of $\phi(\mathcal{H})$ represent these costs. Consider the number of calls to $\operatorname{Visit}(x, I)$ for a particular $\mathcal{H}$ node $x$. According to step 3 of Visit, there will be zero calls to $x$ unless $\operatorname{NORM}(x) \neq$ $\operatorname{NORM}(p(x))$. If it is the case that $\operatorname{NORM}(x) \neq \operatorname{NORM}(p(x))$, then for all recursive calls on $x$, the given interval $I$ will have the same width: $\operatorname{NORM}(z)$ for some ancestor $z$ of $x$. By Definition 4.1(i), $\operatorname{NORM}(z) \geq \operatorname{NORM}(x)$, and therefore the number of such recursive calls on $x$ is $\leq \operatorname{DIAM}(x) / \operatorname{NORM}(x)+2$; the extra 2 counts the first and last recursive calls, which may cover negligible parts of the interval $[d(s, x), d(s, x)+\operatorname{DIAM}(x)]$. By Definition 4.1(iii), $|\mathcal{H}|<2 n$, and therefore the total number of recursive calls is bounded by $4 n+\sum_{x} \operatorname{DIAM}(x) / \operatorname{NORM}(x)$, where the summation is over $\mathcal{H}$ nodes whose NORM-values differ from their parents' NORM-values.

Now consider the bucketing costs of VISIT if implemented with the bucket-heap. According to steps 2 and 3 , a node $y$ is bucketed either because $\operatorname{Visit}(p(y), \cdot)$ was called for the first time, or its parent $p(y)$ was removed from the first open bucket (of some bucket array), say bucket $[a, a+\operatorname{NORM}(p(y)))$. In either case, this means that $d(s, p(y)) \in[a, a+\operatorname{NORM}(p(y)))$ and that $d(s, y) \in[a, a+\operatorname{NORM}(p(y))+\operatorname{DIAM}(p(y)))$. To use the terminology of Lemma 4.3, $\Delta_{y} \leq\lceil\operatorname{DiAm}(p(y)) / \operatorname{NORM}(p(y))\rceil$, and the total bucketing costs would be \#(buckets scanned $)+$ \#(insertions $)+$ \#(dec-keys $)+$ $\sum_{x} \log (\operatorname{DIAM}(p(x)) / \operatorname{NORM}(p(x))+1)$, which is $O(\phi(\mathcal{H})+m+n)$.

In section 5 we give a method for constructing a proper hierarchy $\mathcal{H}$ such that $\phi(\mathcal{H})=O(n)$. This bound together with Lemma 4.4 shows that we can compute SSSP in $O(\operatorname{SPLIT-FIndmin}(m, n))$ time, given a suitable hierarchy. Asymptotically speaking, this bound is the best we are able to achieve. However, the promising experimental results of a simplified version of our algorithm [PRS02] have led us to design an alternate implementation of GENERALIZED-VISIT that is both theoretically fast and easier to code.

4.5. A practical implementation of Generalized-Visit. In this section we present another implementation of Generalized-Visit, called Visit-B. Although VISIT-B is a bit slower than VISIT in the asymptotic sense, it has other advantages. Unlike VISIT, VISIT-B treats all internal hierarchy nodes in the same way and is generally more streamlined. VISIT-B also works with any optimal off-the-shelf priority queue, such as a Fibonacci heap [FT87]. We will prove later that the asymptotic running time of VIsiT-B is $O\left(m+n \log ^{*} n\right)$. Therefore, if $m / n=\Omega\left(\log ^{*} n\right)$, both VISIT and VIsIT-B run in optimal $O(m)$ time.

The pseudocode for VIsiT-B is given as follows. 


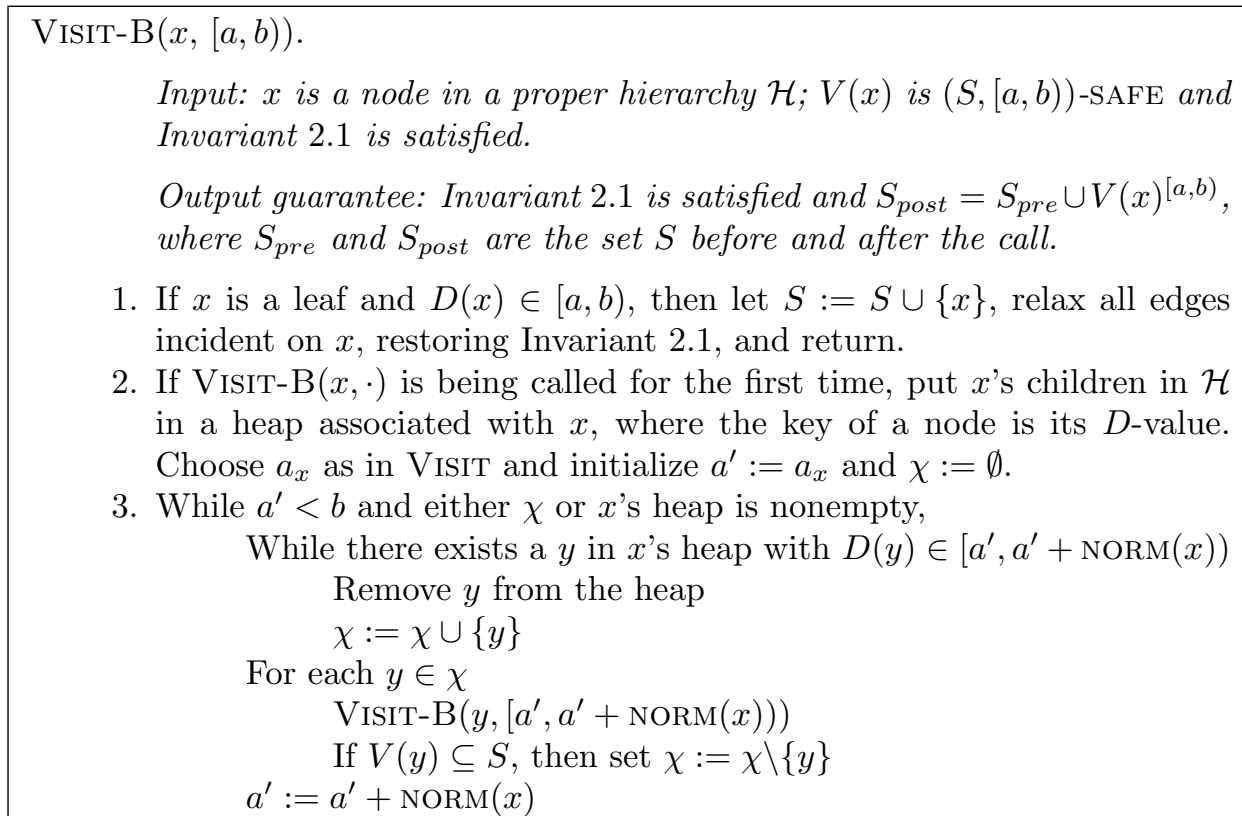

The proof of correctness for VISIT-B follows the same lines as that for Visit. It is easy to establish that before the for-loop in step 3 is executed, $\chi=\{y: p(y)=$ $x, D(y)<a^{\prime}+\operatorname{NORM}(x)$, and $\left.V(y) \nsubseteq S\right\}$, so VISIT-B is actually a more straightforward implementation of Generalized-Visit than Visit. In Visit-B the $\operatorname{NORM}(x)$ partition for $x$ corresponds to $x$ 's children, whereas in VISIT the partition begins with $x$ 's children but is decomposed progressively.

Lemma 4.5. Let $\mathcal{H}$ be a proper hierarchy. Computing SSSPs with VISIT-B on $\mathcal{H}$ takes time $O(\operatorname{Split-Findmin}(m, n)+\psi(\mathcal{H}))$, where $\operatorname{SPLiT-Findmin}(m, n)$ is the complexity of the split-findmin problem and

$$
\psi(\mathcal{H})=\sum_{x \in \mathcal{H}}\left(\frac{\operatorname{DiAm}(x)}{\operatorname{NORM}(x)}+\operatorname{DEG}(x) \log \operatorname{DEG}(x)\right) .
$$

Proof. The SPLIT-FIndmin term plays the same role in Visit-B as in Visit. VISIT-B is different than VISIT in that it makes recursive calls on all hierarchy nodes, not just those with different NORM-values than their parents. Using the same argument as in Lemma 4.5, we can bound the number of recursive calls of the form VISIT$\mathrm{B}(x, \cdot)$ as $\operatorname{DiAm}(x) / \operatorname{NORM}(x)+2$; this gives the first summation in $\psi(\mathcal{H})$. Assuming an optimal heap is used (for example, a Fibonacci heap [FT87]), all decrease-keys take $O(m)$ time, and all deletions take $\sum_{x} \operatorname{DEG}(x) \log \mathrm{DEG}(x)$ time. The bound on deletions follows since each of the $\operatorname{DEG}(x)$ children of $x$ is inserted into and deleted from $x$ 's heap at most once.

In section 5 we construct a hierarchy $\mathcal{H}$ such that $\psi(\mathcal{H})=\Theta\left(n \log ^{*} n\right)$, implying an overall bound on Visit-B of $O\left(m+n \log ^{*} n\right)$, since $\operatorname{SPLIT-FINDMin}(m, n)=$ $O(m \alpha(m, n))=O\left(m+n \log ^{*} n\right)$. Even though $\psi(\mathcal{H})=\Omega\left(n \log ^{*} n\right)$ in the worst case, we are only able to construct very contrived graphs for which this lower bound is tight.

5. Efficient construction of balanced hierarchies. In this section we construct a hierarchy that works well for both VIsIT and Visit-B. The construction procedure has three distinct phases. In phase 1 we find the graph's minimum span- 
ning tree, denoted $M$, and classify its edges by length. This classification immediately induces a coarse hierarchy, denoted $\mathcal{H}_{0}$, which is analogous to the component hierarchy defined by Thorup [Tho99] for integer-weighted graphs. Although $\mathcal{H}_{0}$ is proper, using it to run VISIT or VISIT-B may result in a slow SSSP algorithm. In particular, $\phi\left(\mathcal{H}_{0}\right)$ and $\psi\left(\mathcal{H}_{0}\right)$ can easily be $\Theta(n \log n)$, giving no improvement over Dijkstra's algorithm. Phase 2 facilitates phase 3 , in which we produce a refinement of $\mathcal{H}_{0}$, called $\mathcal{H}$; this is the "well balanced" hierarchy we referred to earlier. The refined hierarchy $\mathcal{H}$ is constructed so as to minimize the $\phi(\mathcal{H})$ and $\psi(\mathcal{H})$ terms in the running times of VISIT and Visit-B. In particular, $\phi(\mathcal{H})$ will be $O(n)$, and $\psi(\mathcal{H})$ will be $O\left(n \log ^{*} n\right)$. Although $\mathcal{H}$ could be constructed directly from $M$ (the graph's minimum spanning tree), we would not be able to prove the time bound of Theorem 1.1 using this method. The purpose of phase 2 is to generate a collection of small auxiliary graphs that-loosely speaking - capture the structure and edge lengths of certain subtrees of the minimum spanning tree. Using the auxiliary graphs in lieu of $M$, we are able to construct $\mathcal{H}$ in phase 3 in $O(n)$ time.

In section 5.1 we define all the notation and properties used in phases 1,2 , and 3 (sections 5.2, 5.3, and 5.4, respectively). In section 5.5 we prove that $\phi(\mathcal{H})=O(n)$ and $\psi(\mathcal{H})=O\left(n \log ^{*} n\right)$.

\subsection{Some definitions and properties.}

5.1.1. The coarse hierarchy. Our refined hierarchy $\mathcal{H}$ is derived from a coarse hierarchy $\mathcal{H}_{0}$, which is defined here and in section 5.2. Although $\mathcal{H}_{0}$ is typically very simple to describe, the general definition of $\mathcal{H}_{0}$ is rather complicated since it must take into account certain extreme circumstances. $\mathcal{H}_{0}$ is defined w.r.t. an increasing sequence of NORM-values: $\mathrm{NORM}_{1}, \mathrm{NORM}_{2}, \ldots$, where all edge lengths are at least as large as $\mathrm{NORM}_{1}$. (Typically $\mathrm{NORM}_{i+1}=2 \cdot \mathrm{NORM}_{i}$; however, this is not true in general.) We will say that an edge $e$ is at level $i$ if $\ell(e) \in\left[\operatorname{NORM}_{i}, \mathrm{NORM}_{i+1}\right)$, or alternatively, we may write $\operatorname{NORM}(e)=\operatorname{NORM}_{i}$ to express that $e$ is at level $i$. A level $i$ subgraph is a maximal connected subgraph restricted to edges with level $i$ or less, that is, with length strictly less than $\mathrm{NORM}_{i+1}$. Therefore, the level zero subgraphs consist of single vertices. A level $i$ node in $\mathcal{H}_{0}$ corresponds to a nonredundant level $i$ subgraph, where a level $i$ subgraph is redundant if it is also a level $i-1$ subgraph. This nonredundancy property guarantees that all nonleaf $\mathcal{H}_{0}$-nodes have at least two children. The ancestor relationship in $\mathcal{H}_{0}$ should be clear: $x$ is an ancestor of $y$ if and only if the subgraph of $y$ is contained in the subgraph of $x$, i.e., $V(y) \subseteq$ $V(x)$. The leaves of $\mathcal{H}_{0}$ naturally correspond to graph vertices, and the internal nodes to subgraphs. The coarse hierarchy $\mathcal{H}_{0}$ clearly satisfies Definition 4.1(i), (iii), (iv); however, we have to be careful in choosing the NORM-values if we want it to be a proper hierarchy, that is, for it to satisfy Definition 4.1(ii) as well. Our method for choosing the NORM-values is deferred to section 5.2.

5.1.2. The minimum spanning tree. By the cut property of minimum spanning trees (see [CLRS01, PR02c]) the $\mathcal{H}_{0}$ w.r.t. $G$ is identical to the $\mathcal{H}_{0}$ w.r.t. $M$, the minimum spanning tree (MST) of $G$. Therefore, the remainder of this section is mainly concerned with $M$, not the graph itself. If $X \subseteq V(G)$ is a set of vertices, we let $M(X)$ be the minimal connected subtree of $M$ containing $X$. Notice that $M(X)$ can include vertices outside of $X$. Later on we will need $M$ to be a rooted tree in order to talk coherently about a vertex's parent, ancestors, children, and so on. Assume that $M$ is rooted at an arbitrary vertex. The notation $\operatorname{Root}(M(X))$ refers to the root of the subtree $M(X)$. 
5.1.3. Mass and diameter. The MASS of a vertex set $X \subseteq V(G)$ is defined as

$$
\operatorname{MASS}(X) \stackrel{\text { def }}{=} \sum_{e \in E(M(X))} \ell(e) .
$$

Extending this notation, we let $M(x)=M(V(x))$ and $\operatorname{MASS}(x)=\operatorname{MASS}(V(x))$, where $x$ is a node in any hierarchy. Since the MST path between two vertices in $M(x)$ is an upper bound on the shortest path between them, $\operatorname{MASS}(x)$ is an upper bound on the diameter of $V(x)$. Recall from Definition 4.1 that DIAM $(x)$ denoted any upper bound on the diameter of $V(x)$; henceforth, we will freely substitute $\operatorname{MASs}(x)$ for $\operatorname{DiAM}(x)$.

5.1.4. Refinement of the coarse hierarchy. We will say that $\mathcal{H}$ is a refinement of $\mathcal{H}_{0}$ if all nodes in $\mathcal{H}_{0}$ are also represented in $\mathcal{H}$. An equivalent definition, which provides us with better imagery, is that $\mathcal{H}$ is derived from $\mathcal{H}_{0}$ by replacing each node $x \in \mathcal{H}_{0}$ with a rooted subhierarchy $H(x)$, where the root of $H(x)$ corresponds to (and is also referred to as) $x$ and the leaves of $H(x)$ correspond to the children of $x$ in $\mathcal{H}_{0}$. Consider a refinement $\mathcal{H}$ of $\mathcal{H}_{0}$ where each internal node $y$ in $H(x)$ satisfies $\operatorname{DEG}(y) \neq 1$ and $\operatorname{NORM}(y)=\operatorname{NORM}(x)$. One can easily verify from Definitions 3.2 and 4.1 that if $\mathcal{H}_{0}$ is a proper hierarchy, so too is $\mathcal{H}$. Of course, in order for $\phi(\mathcal{H})$ and $\psi(\mathcal{H})$ to be linear or near-linear, $H(x)$ must satisfy certain properties. In particular, it must be sufficiently short and balanced. By balanced we mean that a node's mass should not be too much smaller than its parent's mass.

5.1.5. Lambda values. We will use the following $\lambda$-values in order to quantify precisely our notion of balance:

$$
\lambda_{0}=0, \quad \lambda_{1}=12 \quad \text { and } \quad \lambda_{q+1}=2^{\lambda_{q} \cdot 2^{-q}} .
$$

Lemma 5.1 gives a lower bound on the growth of the $\lambda$-values; we give a short proof before moving on.

Lemma 5.1. $\min \left\{q: \lambda_{q} \geq n\right\} \leq 2 \log ^{*} n$.

Proof. Let $\mathcal{S}_{q}$ be a stack of $q$ twos; for example, $\mathcal{S}_{3}=2^{2^{2}}=16$. We will prove that $\lambda_{q} \geq \mathcal{S}_{\lfloor q / 2\rfloor}$, giving the lemma. One can verify that this statement holds for $q \leq 9$. Assume that it holds for all $q^{\prime} \leq q$.

$$
\begin{aligned}
\lambda_{q+1} & =2^{2^{\lambda_{q-1} \cdot 2^{-(q-1)}} 2^{-q}} & \text { \{definition of } \left.\lambda_{q+1}\right\} \\
& \geq 2^{2^{\mathcal{S}_{\lfloor(q-1) / 2\rfloor} 2^{-(q-1)}-q}} & \text { \{inductive assumption }\} \\
& \geq 2^{2^{\mathcal{S}_{\lfloor(q-1) / 2\rfloor-1}}}=\mathcal{S}_{\lfloor(q+1) / 2\rfloor} & \text { \{holds for } q \geq 9\} .
\end{aligned}
$$

The third line follows from the inequality $\mathcal{S}_{\lfloor(q-1) / 2\rfloor} \cdot 2^{-(q-1)}-q \geq \mathcal{S}_{\lfloor(q-1) / 2\rfloor-1}$, which holds for $q \geq 9$.

5.1.6. Ranks. Recall from section 5.1 .4 that our refined hierarchy $\mathcal{H}$ is derived from $\mathcal{H}_{0}$ by replacing each node $x \in \mathcal{H}_{0}$ with a subhierarchy $H(x)$. We assign to all nodes in $H(x)$ a nonnegative integer rank. The analysis of our construction would become very simple if for every rank $j$ node $y$ in $H(x), \operatorname{MASs}(y)=\lambda_{j} \cdot \operatorname{NORM}(x)$. Although this is our ideal situation, the nature of our construction does not allow us to place any nontrivial lower or upper bounds on the mass of $y$. We will assign ranks in order to satisfy Property 5.1, given below, which ensures us a sufficiently 
good approximation to the ideal. It is mainly the internal nodes of $H(x)$ that can have subideal ranks; we assign ranks to the leaves of $H(x)$ (representing children of $x$ in $\mathcal{H}_{0}$ ) to be as close to the ideal as possible.

We should point out that the assignment of ranks is mostly for the purpose of analysis. Rank information is never stored explicitly in the hierarchy nodes, nor is rank information used, implicitly or explicitly, in the computation of shortest paths. We only refer to ranks in the construction of $\mathcal{H}$ and when analyzing their effect on the $\phi$ and $\psi$ functions.

Property 5.1. Let $x \in \mathcal{H}_{0}$ and $y, z \in H(x) \subseteq \mathcal{H}$.

(a) If $y$ is an internal node of $H(x)$, then $\operatorname{NORM}(y)=\operatorname{NORM}(x)$ and $\operatorname{DEG}(y)>1$.

(b) If $y$ is a leaf of $H(x)$ (i.e., a child of $x$ in $\mathcal{H}_{0}$ ), then y has rank $j$, where $j$ is maximal s.t. $\operatorname{MASS}(y) / \operatorname{NORM}(x) \geq \lambda_{j}$.

(c) Let $y$ be a child of a rank $j$ node. We call $y$ stunted if $\operatorname{MASS}(y) / \operatorname{NORM}(x)<$ $\lambda_{j-1} / 2$. Each node has at most one stunted child.

(d) Let $y$ be of rank $j$. The children of $y$ can be divided into three sets: $Y_{1}, Y_{2}$, and a singleton $\{z\}$ such that $\left(\operatorname{MASs}\left(Y_{1}\right)+\operatorname{MASs}\left(Y_{2}\right)\right) / \operatorname{NORM}(x)<(2+o(1)) \cdot \lambda_{j}$.

(e) Let $\mathcal{X}$ be the nodes of $H(x)$ of some specific rank. Then $\sum_{y \in \mathcal{X}} \operatorname{Mass}(y) \leq$ $2 \cdot \operatorname{MASS}(x)$.

Before moving on, let us examine some features of Property 5.1. Part (a) is asserted to guarantee that $\mathcal{H}$ is proper. Part (b) shows how we set the rank of leaves of $H(x)$. Part (c) says that at most one child of any node is less than half its ideal mass. Part (d) is a little technical but basically says that for a rank $j$ node $y$, although $\operatorname{MAss}(y)$ may be huge, the children of $y$ can be divided into sets $Y_{1}, Y_{2},\{z\}$ such that $Y_{1}$ and $Y_{2}$ are of reasonable mass - around $\lambda_{j} \cdot \operatorname{NORM}(x)$. However, no bound is placed on the mass contributed by $z$. Part (e) says that if we restrict our attention to the nodes of a particular rank, their subgraphs do not overlap in too many places. To see how two subgraphs might overlap, consider $\left\{x_{i}\right\}$, the set of nodes of some rank in $H(x)$. By our construction it will always be the case that the vertex sets $\left\{V\left(x_{i}\right)\right\}$ are disjoint; however, this does not imply that the subtrees $\left\{M\left(x_{i}\right)\right\}$ are edge-disjoint because $M\left(x_{i}\right)$ can, in general, be much larger than $V\left(x_{i}\right)$.

We show in section 5.5 that if $\mathcal{H}$ is a refinement of $\mathcal{H}_{0}$ and $\mathcal{H}$ satisfies Property 5.1 , then $\phi(\mathcal{H})=O(n)$ and $\psi(\mathcal{H})=O\left(n \log ^{*} n\right)$. Recall from Lemmas 4.4 and 4.5 that $\phi(\mathcal{H})$ and $\psi(\mathcal{H})$ are terms in the running times of VISIT and VISIT-B, respectively.

5.2. Phase 1: The MST and the coarse hierarchy. Pettie and Ramachandran [PR02c] recently gave an MST algorithm that runs in time proportional to the decision-tree complexity of the MST problem. As the complexity of MST is trivially $\Omega(m)$ and only known to be $O(m \alpha(m, n))$ [Chaz00], it is unknown whether this cost will dominate or be dominated by the $\operatorname{SPLIT-FIndmin}(m, n)$ term. (This issue is mainly of theoretical interest.) In the analysis we use $\operatorname{MST}(m, n)$ to denote the cost of computing the MST. This may be interpreted as the decision-tree complexity of MST [PR02c] or the randomized complexity of MST, which is known to be linear [KKT95, PR02b].

Recall from section 5.1.1 that $\mathcal{H}_{0}$ was defined w.r.t. an arbitrary increasing sequence of NORM-values. We describe below exactly how the NORM-values are chosen, then prove that $\mathcal{H}_{0}$ is a proper hierarchy. Our method depends on how large $r$ is, which is the ratio of the maximum-to-minimum edge length in the minimum spanning tree. If $r<2^{n}$, which can easily be determined in $O(n)$ time, then the possible NORMvalues are $\left\{\ell_{\min } \cdot 2^{i}: 0 \leq i \leq \log r+1\right\}$, where $\ell_{\min }$ is the minimum edge length in the graph. If $r \geq 2^{n}$, then let $e_{1}, \ldots, e_{n-1}$ be the edges in $M$ in nondecreasing 
order by length and let $J=\{1\} \cup\left\{j: \ell\left(e_{j}\right)>n \cdot \ell\left(e_{j-1}\right)\right\}$ be the indices that mark large separations in the $\left(\ell\left(e_{i}\right)\right)_{1 \leq i<n}$ sequence. The possible NORM-values are then $\left\{\ell\left(e_{j}\right) \cdot 2^{i}: i \geq 0, j \in J\right.$ and $\left.\ell\left(e_{j}\right) \cdot 2^{i}<\ell\left(e_{j+1}\right)\right\}$.

Under either definition, $\mathrm{NORM}_{i}$ is the $i$ th largest NORM-value, and for an edge $e \in E(M), \operatorname{NORM}(e)=\operatorname{NORM}_{i}$ if $\ell(e) \in\left[\mathrm{NORM}_{i}, \mathrm{NORM}_{i+1}\right)$. Notice that if no edge length falls within the interval $\left[\mathrm{NORM}_{i}, \mathrm{NORM}_{i+1}\right)$, then $\mathrm{NORM}_{i}$ is an unused NORMvalue. We only need to keep track of the NORM-values in use, of which there are no more than $n-1$.

Lemma 5.2. The coarse hierarchy $\mathcal{H}_{0}$ is a proper hierarchy.

Proof. As we observed before, parts (i), (iii), and (iv) of Definition 4.1 are satisfied for any monotonically increasing sequence of NORM-values. Definition 4.1(ii) states that if $x$ is a hierarchy node, either $\operatorname{NORM}(p(x)) / \operatorname{NORM}(x)$ is an integer or $\operatorname{DIAM}(x) / \operatorname{NORM}(p(x))<1$. Suppose that $x$ is a hierarchy node and $\operatorname{NORM}(p(x)) / \operatorname{NORM}(x)$ is not integral; then $\operatorname{NORM}(x)=\ell\left(e_{j_{1}}\right) \cdot 2^{i_{1}}$ and $\operatorname{NORM}(p(x))=\ell\left(e_{j_{2}}\right) \cdot 2^{i_{2}}$, where $j_{2}>j_{1}$. By our method for choosing the NORM-values, the lengths of all MST edges are either at least $\ell\left(e_{j_{2}}\right)$ or less than $\ell\left(e_{j_{2}}\right) / n$. Since edges in $M(x)$ have length less than $\ell\left(e_{j_{2}}\right)$, and hence less than $\ell\left(e_{j_{2}}\right) / n$, DIAM $(x)<(|V(x)|-1) \cdot \ell\left(e_{j_{2}}\right) / n<\ell\left(e_{j_{2}}\right) \leq$ $\operatorname{NORM}(p(x))$.

LEMma 5.3. We can compute the minimum spanning tree $M$, and $\operatorname{NORM}(e)$ for all $e \in E(M)$, in $O(\operatorname{MST}(m, n)+\min \{n \log \log r, n \log n\})$ time.

Proof. $\operatorname{MST}(m, n)$ represents the time to find $M$. If $r<2^{n}$, then by Lemma 2.2 we can find $\operatorname{NORM}(e)$ for all $e \in M$ in $O(\log r+n \log \log r)=O(n \log \log r)$ time. If $r \geq 2^{n}$, then $n \log \log r=\Omega(n \log n)$, so we simply sort the edges of $M$ and determine the indices $J$ in $O(n \log n)$ time. Suppose there are $n_{j}$ edges $e$ s.t. $\operatorname{NORM}(e)$ is of the form $\ell\left(e_{j}\right) \cdot 2^{i}$. Since $\ell(e) / \ell\left(e_{j}\right) \leq n^{n_{j}}$, we need only generate $n_{j} \log n$ values of the form $\ell\left(e_{j}\right) \cdot 2^{i}$. A list of the $\sum_{j} n_{j} \log n=n \log n$ possible NORM-values can easily be generated in sorted order. By merging this list with the list of MST edge lengths, we can determine $\operatorname{NoRm}(e)$ for all $e \in M$ in $O(n \log n)$ time.

Lemma 5.4, given below, will come in handy in bounding the running time of our preprocessing and SSSP algorithms. It says that the total normalized mass in $\mathcal{H}_{0}$ is linear in $n$. Variations of Lemma 5.4 are at the core of the hierarchy approach [Tho99, Hag00, Pet04, Pet02b].

LEMMa 5.4.

$$
\sum_{x \in \mathcal{H}_{0}} \frac{\operatorname{MASS}(x)}{\operatorname{NORM}(x)}<4(n-1)
$$

Proof. Recall that the notation $\operatorname{NORM}(e)=\mathrm{NORM}_{i}$ stands for $\ell(e) \in$ $\left[\mathrm{NORM}_{i}, \mathrm{NORM}_{i+1}\right)$, where $\mathrm{NORM}_{i}$, is the $i$ th largest NORM-value. Observe that if $e \in M$ is an MST edge with $\operatorname{NORM}(e)=\operatorname{NORM}_{i}, e$ can be included in $\operatorname{Mass}(x)$ for no more than one $x$ at levels $i, i+1, \ldots$ in $\mathcal{H}_{0}$. Also, it follows from our definitions that for every $\mathrm{NORM}_{i}$ in use, $\operatorname{NORM}_{i+1} / \mathrm{NORM}_{i} \geq 2$, and for any MST edge, $\ell(e) / \operatorname{NORM}(e)<2$. Therefore, we can bound the normalized mass in $\mathcal{H}_{0}$ as

$$
\begin{aligned}
\sum_{x \in \mathcal{H}_{0}} \frac{\operatorname{MASs}(x)}{\operatorname{NORM}(x)} & \leq \sum_{\substack{e \in M \\
\operatorname{NORM}(e)=\mathrm{NORM}_{i}}} \sum_{j=i}^{\infty} \frac{\ell(e)}{\operatorname{NORM}_{j}} \\
& \leq \sum_{\substack{e \in M \\
\operatorname{NORM}(e)=\mathrm{NORM}_{i}}} \sum_{j=i}^{\infty} \frac{\ell(e)}{2^{j-i} \cdot \operatorname{NORM}_{i}}<4(n-1) .
\end{aligned}
$$




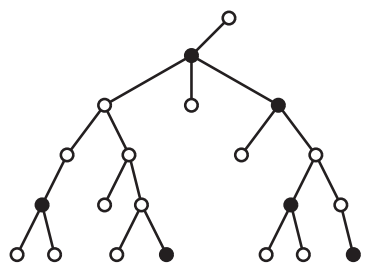

Black vertices are in $X$

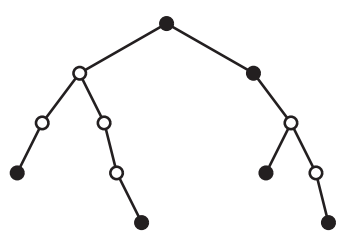

$M(X)$

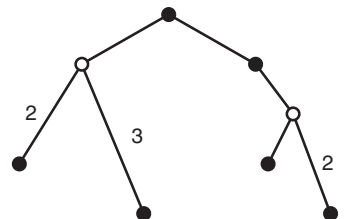

$T(X)$

FIG. 2. On the left is a subtree of $M$, the $M S T$, where $X$ is the set of blackened vertices. In the center is $M(X)$, the minimal subtree of $M$ connecting $X$, and on the right is $T(X)$, derived from $M(X)$ by splicing out unblackened degree 2 nodes in $M(X)$ and adjusting edge lengths appropriately. Unless otherwise marked, all edges in this example are of length 1.

Implicit in Lemma 5.4 is a simple accounting scheme where we treat mass, or more accurately normalized mass, as a currency equivalent to computational work. A hierarchy node $x$ "owns" $\operatorname{MASS}(x) / \operatorname{NORM}(x)$ units of currency. If we can then show that the share of some computation relating to $x$ is bounded by $k$ times its currency, the total time for this computation is $O(k n)$, that is, of course, if all computation is attributable to some hierarchy node. Although simple, this accounting scheme is very powerful and can become quite involved [Pet04, Pet02b, Pet03].

5.3. Phase 2: Constructing $\boldsymbol{T}(\boldsymbol{x})$ trees. Although it is possible to construct an $H(x)$ that satisfies Property 5.1 by working directly with the subtree $M(x)$, we are unable to efficiently compute $H(x)$ in this way. The problem is that we have time roughly proportional to the size of $H(x)$ to construct $H(x)$, whereas $M(x)$ could be significantly larger than $H(x)$. Our solution is to construct a succinct tree $T(x)$ that preserves the essential structure of $M(x)$ while having size roughly the same as $H(x)$.

For $X \subseteq V(G)$, let $T(X)$ be the subtree derived from $M(X)$ by splicing out all single-child vertices in $V(M(X))-X$. That is, we replace each chain of vertices in $M(X)$, where only the end vertices are potentially in $X$, with a single edge; the length of this edge is the sum of its corresponding edge lengths in $M(X)$. Since there is a correspondence between vertices in $T(X)$ and $M$, we will refer to $T(X)$ vertices by their names in $M$. Figure 2 gives examples of $M(X)$ and $T(X)$ trees, where $X$ is the set of blackened vertices.

If $x \in \mathcal{H}_{0}$ and $\left\{x_{j}\right\}_{j}$ is the set of children of $x$, then let $T(x)$ be the tree $T\left(\left\{\operatorname{Root}\left(M\left(x_{j}\right)\right)\right\}_{j}\right)$; note that $\operatorname{Root}(M(x))$ is included in $\left\{\operatorname{Root}\left(M\left(x_{j}\right)\right)\right\}_{j}$. Since only some of the edges of $M(x)$ are represented in $T(x)$, it is possible that the total length of $T(x)$ is significantly less than the total length of $M(x)$ (the MASS of $M(x)$ ); however, we will require that any subgraph of $T(x)$ have roughly the same mass as an equivalent subgraph in $M(x)$. In order to accomplish this we attribute certain amounts of mass to the vertices of $T(x)$ as follows. Suppose that $y$ is a child of $x$ in $\mathcal{H}_{0}$ and $v=\operatorname{ROOT}(y)$ is the corresponding root vertex in $T(x)$. We let $\operatorname{MAss}(v)=\operatorname{MASs}(y)$. All other vertices in $T(x)$ have zero mass. The mass of a subtree of $T(x)$ is then the sum of its edge lengths plus the collective mass of its vertices.

We will think of a subtree of $T(x)$ as corresponding to a subtree of $M(x)$. Each edge in $T(x)$ corresponds naturally to a path in $M(x)$, and each vertex in $T(x)$ with nonzero mass corresponds to a subtree of $M(x)$.

LEMMA 5.5. For $x \in \mathcal{H}_{0}$,

(i) $\operatorname{DEG}(x) \leq|V(T(x))|<2 \cdot \operatorname{DEG}(x)$;

(ii) let $T_{1}$ be a subtree of $T(x)$ and $T_{2}$ be the corresponding tree in $M(x)$. Then 

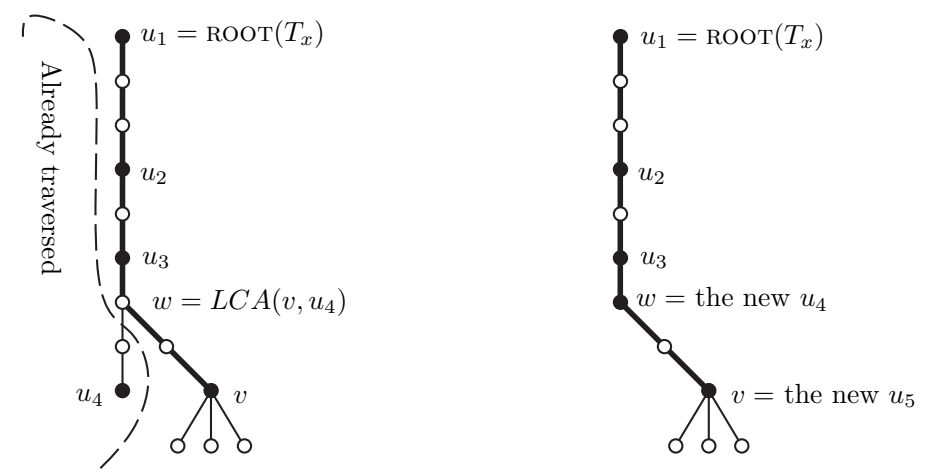

FIG. 3. The blackened vertices represent those known to be in $T(x)$. The active path of the traversal is shown in bold edges. Before $v$ is processed (left) the stack consists of $\left\langle u_{1}, u_{2}, u_{3}, u_{4}\right\rangle$, where $u_{4}$ is the last vertex in the traversal known to be in $T(x)$ and $w=L C A\left(v, u_{4}\right)$, which implies $w \in T(x)$. After $v$ is processed (right) the stack is set to $\left\langle u_{1}, u_{2}, u_{3}, w, v\right\rangle$ and $w$ is blackened.

$$
\operatorname{MASS}\left(T_{2}\right) \leq \operatorname{MASS}\left(T_{1}\right) \leq 2 \cdot \operatorname{MASS}\left(T_{2}\right) .
$$

Proof. Part (i) follows from two observations. First, $T(x)$ has no degree two vertices. Second, there are at most $\operatorname{DEG}(x)$ leaves of $T(x)$ since each such leaf corresponds to a vertex $\operatorname{RoOT}(M(y))$ for some child $y$ of $x$ in $\mathcal{H}_{0}$. Part (ii) follows since all mass in $T_{2}$ is represented in $T_{1}$, and each edge in $T_{2}$ contributes to the mass of at most one edge and one vertex in $T_{1}$.

We construct $T(x)$ with a kind of depth first traversal of the minimum spanning tree, using the procedure Succinct-Tree, given below. Succinct-Tree focuses on some fixed $\mathcal{H}_{0}$-node $x$. We will explain how Succinct-TreE works with the aid of the diagram in Figure 3. At every point in the traversal we maintain a stack of vertices $\left\langle u_{1}, \ldots, u_{q}\right\rangle$ consisting of all vertices known to be in $T(x)$, whose parents in $T(x)$ are not yet fixed. The stack has the following properties: $u_{i}$ is ancestral to $u_{i+1}$, $\left\langle u_{1}, \ldots, u_{q-1}\right\rangle$ are on the active path of the traversal, and $u_{q}$ is the last vertex known to be in $T(x)$ encountered in the traversal.

In Figure 3 the stack consists of $\left\langle u_{1}, \ldots, u_{4}\right\rangle$, where $\left\langle u_{1}, u_{2}, u_{3}\right\rangle$ are on the active path of the traversal, marked in bold edges. The preprocessing of $v$ (before making recursive calls) is to do nothing if $v \notin\left\{\operatorname{ROOT}\left(M\left(x_{j}\right)\right)\right\}_{j}$. Otherwise, we update the stack to reflect our new knowledge about the edges and vertices of $T(x)$. The vertex $w=L C A\left(u_{q}, v\right)=L C A\left(u_{4}, v\right)$ must be in $T(x)$. There are three cases: either $w$ is the ultimate or penultimate vertex in the stack $\left(u_{q}\right.$ or $\left.u_{q-1}\right)$, that is, we already know $w \in T(x)$, or $w$ lies somewhere on the path between $u_{q}$ and $u_{q-1}$. Figure 3 diagrams the third situation. Because no $T(x)$ vertices were encountered in the traversal between $u_{q}=u_{4}$ and $v$, there can be no new $T(x)$ vertices discovered on the path between $u_{q}$ and $w$. Therefore, we can pop $u_{q}$ off the stack, designating its parent in $T(x)$ to be $w$, and push $w$ and $v$ onto the stack. The other two situations, when $w=u_{q}$ or $w=u_{q-1}$, are simpler. If $w=u_{q}$, then we simply push $v$ onto the stack, and if $w=u_{q-1}$, we pop $u_{q}$ off the stack and push $v$ on. Now consider the postprocessing of $v$ (performed after all recursive calls), and let $u_{q-1}, u_{q}$ be the last two vertices in the stack. Suppose that $v=u_{q-1}$. We cannot simply do nothing, because when the active path retracts there will be two stack vertices $\left(v=u_{q-1}\right.$ and $u_{q}$ ) outside of the active path, contrary to the stack properties. However, because no $T(x)$ vertices were discovered between $u_{q}$ and $u_{q-1}$, we can safely say that $u_{q-1}$ is the 
parent of $u_{q}$ in $T(x)$. So, to maintain the stack properties, we pop off $u_{q}$ and add the edge $\left(u_{q}, u_{q-1}\right)$ to $T(x)$.

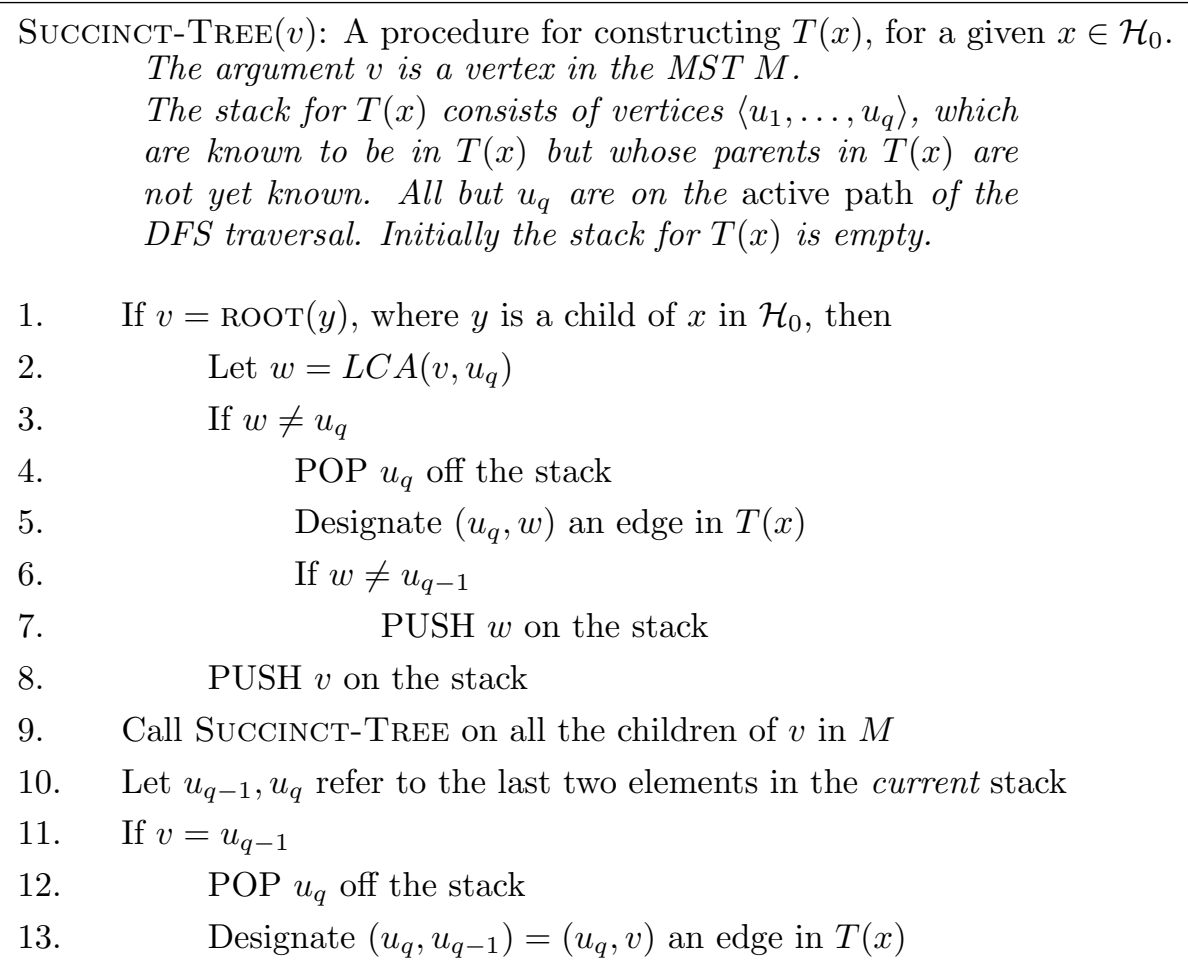

Lemma 5.6. Given the MST and a list of its edges ordered by level, $\mathcal{H}_{0}$ and $\{T(x)\}_{x}$ can be constructed in $O(n)$ time.

Proof. We construct $\mathcal{H}_{0}$ with a union-find structure and mark all vertices in $M$ as roots of $M(x)$ for (one or more) $x \in \mathcal{H}_{0}$. It is easy to see that we can construct all $T(x)$ for $x \in \mathcal{H}_{0}$, with one tree traversal given in procedure Succinct-TreE. We simply maintain a different stack for each $T_{x}$ under construction. Thus if $v$ is the root of several $M\left(y_{1}\right), M\left(y_{2}\right), \ldots$, where $y_{i} \in \mathcal{H}_{0}$, we simply reexecute lines 1-8 and 10-13 of Succinct-Tree for each of $v$ 's roles. Using a well-known union-findbased least common ancestors (LCA) algorithm [AHU76, Tar79b], we can compute the LCAs in line 2 in $O(n \alpha(n))$ time, since the number of finds is linear in the number of nodes in $\mathcal{H}_{0}$. If we use the scheme of Buchsbaum et al. [BKRW98] instead, the cost of finding LCAs is linear; however, since this algorithm is offline (it does not handle LCA queries in the middle of a tree traversal, unlike [AHU76, Tar79b]), we would need to determine what the LCA queries are with an initial pass over the tree. Finally, we compute the length function in $T(x)$ as follows. If $(u, v) \in E(T(x))$ and $v$ is ancestral to $u$ in $M$, then $\ell(u, v)=d_{M}(u, \operatorname{ROOT}(M))-d_{M}(v, \operatorname{ROOT}(M))$, where $d_{M}$ is the distance function for $M$. Clearly the $d_{M}(\cdot, \operatorname{ROOT}(M))$ function can be computed in $O(n)$ time. See Lemma 2.1 for a simulation of subtraction in the comparison-addition model.

5.4. Phase 3: Constructing the refined hierarchy. We show in this section how to construct an $H(x)$ from $T(x)$ that is consistent with Property 5.1.

The REFINE-HieRARCHY procedure, given as pseudocode below, constructs $H(x)$ in a bottom-up fashion by traversing the tree $T(x)$. A call to $\operatorname{Refine-Hierarchy}(v)$, 
where $v \in T(x)$, will produce an array of sets $v[\cdot]$ whose elements are nodes in $H(x)$ that represent (collectively) the subtree of $T(x)$ rooted at $v$. The set $v[j]$ holds rank $j$ nodes, which, taken together, are not yet massive enough to become a rank $j+1$ node. We extend the mass notation to sets $v[\cdot]$ as follows. Bear in mind that this mass is w.r.t. the tree $T(x)$, not $M(x)$. By Lemma 5.5(ii), mass w.r.t. $T(x)$ is a good approximation to the mass of the equivalent subtree in $M(x)$ :

$$
\operatorname{MASS}(v[j])=\operatorname{MASS}\left(\bigcup_{j^{\prime} \leq j} \bigcup_{y \in v\left[j^{\prime}\right]} V(y)\right) .
$$

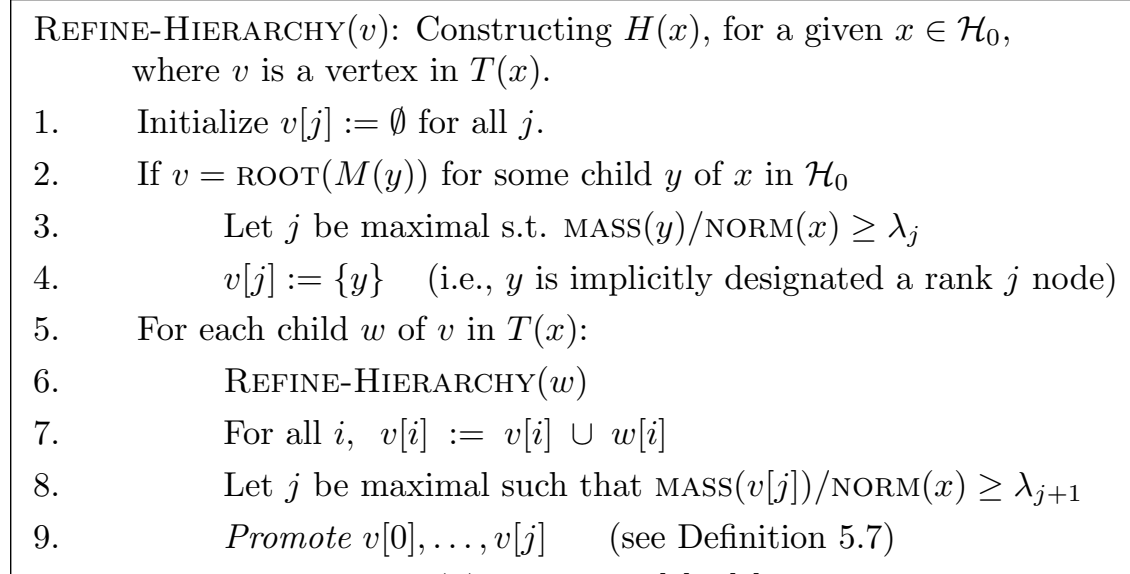

10. If $v$ is the root of $T(x)$, promote $v[0], v[1], \ldots$ until one node remains. (This final node is the root of $H(x)$.)

The structure of REFINE-HIERARCHY is fairly simple. To begin with, we initialize $v[\cdot]$ to be an array of empty sets. Then, if $v$ is a root vertex of a child $y$ of $x$ in $\mathcal{H}_{0}$, we create a node representing $y$ and put it in the proper set in $v[\cdot]$; which set receives $y$ depends only on $\operatorname{Mass}(y)$. Next we process the children of $v$. On each pass through the loop, we pick an as yet unprocessed child $w$ of $v$; recurse on $w$, producing sets $w[\cdot]$ representing the subtree rooted at $w$; and then merge the sets $w[\cdot]$ into their counterparts in $v[\cdot]$. At this point, the mass of some sets may be beyond a critical threshold: the threshold for $v[j]$ is $\lambda_{j+1} \cdot \operatorname{NORM}(x)$. In order to restore a quiescent state in the sets $v[\cdot]$ we perform promotions until no set's mass is above threshold.

Definition 5.7. Promoting the set $v[j]$ involves removing the nodes from $v[j]$, making them the children of a new rank $j+1$ node, and then placing this node in $v[j+1]$. There is one exception: if $|v[j]|=1$, then to comply with Definition 4.1(iii), we simply move the node from $v[j]$ to $v[j+1]$. Promoting the sets $v[0], v[1], \ldots, v[j]$ means promoting $v[0]$, then $v[1]$, up to $v[j]$, in that order.

Suppose that after merging $w[\cdot]$ into $v[\cdot], j$ is maximal such that $\operatorname{Mass}(v[j])$ is beyond its threshold of $\lambda_{j+1} \cdot \operatorname{NORM}(x)$ (there need not be such a $j$ ). We promote the sets $v[0], \ldots, v[j]$, which has the effect of emptying $v[0], \ldots, v[j]$ and adding a new node to $v[j+1]$ representing the nodes formerly in $v[0], \ldots, v[j]$. Lemma 5.8, given below, shows that we can compute the $H(x)$ trees in linear time.

Lemma 5.8. Given $\{T(x)\}_{x},\{H(x)\}_{x}$ can be constructed to satisfy Property 5.1 in $O(n)$ time.

Proof. We first argue that Refine-Hierarchy produces a refinement $\mathcal{H}$ of $\mathcal{H}_{0}$ that satisfies Property 5.1. We then look at how to implement it in linear time. 
Property 5.1(a) states that internal nodes in $H(x)$ must have NORM-values equal to that of $x$, which we satisfy by simply assigning them the proper NORM-values, and that no node of $H(x)$ has one child. By our treatment of one-element sets in the promotion procedure of Definition 5.7, it is simply impossible to create a one-child node in $H(x)$. Property 5.1(e) follows from Lemma 5.5(ii) and the observation that the mass (in $T(x)$ ) represented by nodes of the same rank is disjoint. Now consider Property 5.1(c), regarding stunted nodes. We show that whenever a set $v[j]$ accepts a new node $z$, either $v[j]$ is immediately promoted, or $z$ is not stunted, or the promotion of $z$ into $v[j]$ represents the last promotion in the construction of $H(x)$. Consider the pattern of promotions in line 9 . We promote the sets $v[0], \ldots, v[j]$ in a cascading fashion: $v[0]$ to $v[1], v[1]$ to $v[2]$, and so on. The only set accepting a new node that is not immediately promoted is $v[j+1]$, so in order to prove Property $5.1(\mathrm{c})$ we must show that the node derived from promoting $v[0], \ldots, v[j]$ is not stunted. By choice of $j, \operatorname{MaSs}(v[j]) \geq \lambda_{j+1} \cdot \operatorname{NORM}(x)$, where mass is w.r.t. the tree $T(x)$. By Lemma 5.5 (ii) the mass of the equivalent tree in $M(x)$ is at least $\lambda_{j+1} \cdot \operatorname{NORM}(x) / 2$, which is exactly the threshold for this node being stunted. Finally, consider Property 5.1(d). Before the merging step in line 7, none of the sets in $v[\cdot]$ or $w[\cdot]$ is massive enough to be promoted. Let $v[\cdot]$ and $w[\cdot]$ denote the sets associated with $v$ and $w$ before the merging in step 7 , and let $v^{\prime}[\cdot]$ denote the set associated with $v$ after step 7 . By the definition of MASS we have

$$
\operatorname{MASS}\left(v^{\prime}[j]\right)=\operatorname{MASS}(v[j])+\operatorname{MASS}(w[j])+\ell(v, w)<2 \cdot \lambda_{j+1} \cdot \operatorname{NORM}(x)+\ell(v, w) .
$$

Since $(v, w)$ is an edge in $T(x)$, it can be arbitrarily large compared to $\operatorname{NORM}(x)$, meaning we cannot place any reasonable bound on $\operatorname{MASs}\left(v^{\prime}[j]\right)$ after the merging step. Let us consider how Property 5.1(d) is maintained. Suppose that $v^{\prime}[j]$ is promoted in lines 9 or 10, and let $y$ be the resulting rank $j+1$ node. Using the terminology from Property 5.1(d), let $Y_{1}=v[j], Y_{2}=w[j]$ and let $z$ be the node derived by promoting $v^{\prime}[0], \ldots, v^{\prime}[j-1]$. Since neither $v[j]$ nor $w[j]$ was sufficiently massive to be promoted before they were merged, we have $\left(\operatorname{MASS}\left(Y_{1}\right)+\operatorname{MASS}\left(Y_{2}\right)\right) / \operatorname{NORM}(x)<2 \lambda_{j+1}$. This is slightly stronger than what Property 5.1(d) calls for, which is the inequality < $(2+o(1)) \lambda_{j+1}$. We'll see why the $(2+o(1))$ is needed below.

Suppose that we implemented REFINE-HIERARCHY in a straightforward manner. Let $L$ be the (known) maximum possible index of any nonempty set $v[\cdot]$ during the course of ReFine-HieraRCHY. One can easily see that the initialization in lines 1-4 takes $O(L+1)$ time and that, exclusive of recursive calls, each time through the forloop in line 5 takes $O(L+1)$ amortized time. (The bound on line 5 is amortized since promoting a set $v[j]$ takes worst case $O(|v[j]|+1)$ time but only constant amortized time.) The only hidden costs in this procedure are updating the MASS of sets, which is done as follows. After the merging step in line 7, we simply set $\operatorname{mass}(v[j]):=$ $\operatorname{MASS}(v[j])+\ell(v, w)+\operatorname{MASS}(w[j])$ for each $j \leq L$. Therefore the total cost of computing $H(x)$ from $T(x)$ is $O((L+1) \cdot|T(x)|)$. We can bound $L$ as $L \leq 2 \log ^{*}(4 n)$ as follows. The first node placed in any previously empty set is unstunted; therefore, by Lemma 5.1, the maximum nonempty set has rank at most $2 \log ^{*}(\operatorname{MASS}(T(x)) / \operatorname{NORM}(x))$. By Lemma 5.5(ii) and the construction of $\mathcal{H}_{0}, \operatorname{MASS}(T(x)) \leq 2 \cdot \operatorname{MASs}(M(x))<4(n-1)$. $\operatorname{NORM}(x)$.

In order to reduce the cost to linear we make a couple adjustments to the REFINEHiERARCHY procedure. First, $v[\cdot]$ is represented as a linked list of nonempty sets. Second, we update the mass variables in a lazy fashion. The time for steps $1-4$ is dominated by the time to find the appropriate $j$ in step 3 , which takes time $t_{1}$ - see 
below. The time for merging the $v[\cdot]$ and $w[\cdot]$ sets in line 7 is only proportional to the shorter list; this time bound is given by expression $t_{2}$ below.

$$
\begin{gathered}
t_{1}=O\left(1+\log ^{*} \frac{\operatorname{MAss}(v)}{\operatorname{NORM}(x)}\right), \\
t_{2}=O\left(1+\log ^{*} \frac{\min \{\operatorname{Mass}(v[\cdot]), \operatorname{MAss}(w[\cdot])\}}{\operatorname{NORM}(x)}\right),
\end{gathered}
$$

where $\operatorname{Mass}(v[\cdot])$ is just the total mass represented by the $v[\cdot]$ sets. We update the mass of only the first $t_{1}+t_{2}$ sets in $v[\cdot]$, and, as a rule, we update $v[j+1]$ half as often as $v[j]$. It is routine to show that REFINE-HIERARCHY will have a lower bound on the mass of $v[j]$ that is off by a $1+\mathrm{o}(1)$ factor, where the $o(1)$ is a function of $j .{ }^{7}$ This leads to the conspicuous $2+o(1)$ in Property 5.1(d). To bound the cost of REFINE-HIERARCHY we model its computation as a binary tree: leaves represent the creation of nodes in lines 1-4, and internal nodes represent the merging events in line 7. The cost of a leaf $f$ is $\log ^{*}(\operatorname{Mass}(f) / \operatorname{NORM}(x))$, and the cost of an internal node $f$ with children $f_{1}$ and $f_{2}$ is $1+\log ^{*}\left(\min \left\{\operatorname{MASS}\left(f_{1}\right) / \operatorname{NORM}(x), \operatorname{MASs}\left(f_{2}\right) / \operatorname{NORM}(x)\right\}\right)$. We can think of charging the cost of $f$ collectively to the mass in the subtree of $f_{1}$ or $f_{2}$, whichever is smaller. Therefore, no unit of mass can be charged for two nodes $f$ and $g$ if the total mass under $f$ is within twice the total mass under $g$. The total cost is then

$$
\sum_{f} \operatorname{cost}(f)=O\left(|T(x)|+\frac{\operatorname{MASS}(T(x))}{\operatorname{NORM}(x)} \cdot \sum_{i=0}^{\infty} \frac{\log ^{*}\left(2^{i}\right)}{2^{i}}\right)=O\left(\frac{\operatorname{MASS}(x)}{\operatorname{NORM}(x)}\right) .
$$

The last equality follows because $|T(x)|=O(\operatorname{MAss}(T(x)) / \operatorname{NORM}(x))=$ $O(\operatorname{Mass}(M(x)) / \operatorname{NORM}(x))$. Summing over all $x \in \mathcal{H}_{0}$, the total cost of constructing $\{H(x)\}_{x \in \mathcal{H}_{0}}$ is, by Lemma $5.4, O(n)$.

LEMma 5.9. In $O(\operatorname{MST}(m, n)+\min \{n \log \log r, n \log n\})$ time we can construct both the coarse hierarchy $\mathcal{H}_{0}$ and a refinement $\mathcal{H}$ of $\mathcal{H}_{0}$ satisfying Property 5.1.

Proof. The proof follows from Lemmas 5.3, 5.6, and 5.8.

5.5. Analysis. In this section we prove bounds on the running times of VISIT and VISIT-B, given an appropriate refined hierarchy such as the one constructed in section 5.4. Theorem 1.1 follows directly from Lemma 5.10, given below, and Lemma 5.9 .

Lemma 5.10. Let $\mathcal{H}$ be any refinement of $\mathcal{H}_{0}$ satisfying Property 5.1. Using $\mathcal{H}$, Visit computes $S S S P$ in $O(\operatorname{SPLIT-FINDMin}(m, n))$ time, and VISIT-B computes SSSP in $O\left(m+n \log ^{*} n\right)$ time.

Proof. We prove that $\phi(\mathcal{H})=O(n)$ and $\psi(\mathcal{H})=O\left(n \log ^{*} n\right)$. Together with Lemmas 4.4 and 4.5, this will complete the proof.

With the observation that $\operatorname{MASS}(x)$ is an upper bound on the diameter of $V(x)$, we will substitute $\operatorname{Mass}(x)$ for $\operatorname{DiAm}(x)$ in the functions $\phi$ and $\psi$. By Lemma 5.4, the first sum in $\phi$ is $O(n)$. The first sum of $\psi(\mathcal{H})$ is much like that in $\phi$, except we sum over all nodes in $\mathcal{H}$, not just those nodes that also appear in $\mathcal{H}_{0}$. By Property 5.1(a), (c), and (d) and Lemma 5.1, the maximum rank of any node in $H(x)$ is

\footnotetext{
${ }^{7}$ The proof of this is somewhat tedious. Basically one shows that for $i<j$ the mass of $v[i]$ can be updated at most $2^{j-i}-1$ times before the mass of $v[j]$ is updated. Since $2^{j-1}-1 \cdot \lambda_{i} \ll \lambda_{j}$, our neglecting to update the mass of $v[j]$ causes a negligible error.
} 
$2 \log ^{*}(\operatorname{Mass}(x) / \operatorname{NORM}(x)) \leq 2 \log ^{*} n$. By Property $5.1(\mathrm{e})$ the total mass of nodes of one rank in $H(x)$ is bounded by $2 \cdot \operatorname{MAss}(x)$. Therefore, we can bound the first sum in $\psi(\mathcal{H})$ as $\sum_{x} \operatorname{MASS}(x) / \operatorname{NORM}(x) \leq 4 \log ^{*} n \cdot \sum_{x \in \mathcal{H}_{0}} \operatorname{MASS}(x) / \operatorname{NORM}(x)$, which is $O\left(n \log ^{*} n\right)$ by Lemma 5.4 .

We now turn to the second summations in $\phi(\mathcal{H})$ and $\psi(\mathcal{H})$, which can be written as $\sum_{x} \operatorname{DEG}(x) \log (\operatorname{MAsS}(x) / \operatorname{NORM}(x))$ and $\sum_{x} \operatorname{DEG}(x) \log \operatorname{DEG}(x)$, respectively. Since $\operatorname{DEG}(x) \leq 1+\operatorname{MASS}(x) / \operatorname{NORM}(x)$, any bound established on the first summation will extend to the second.

Let $y$ be a rank $j$ node. Using the terms from Property 5.1(d), let $\alpha=\left(\operatorname{Mass}\left(Y_{1}\right)+\right.$ $\left.\operatorname{MASS}\left(Y_{2}\right)\right) / \operatorname{NORM}(y)$ and $\beta=\operatorname{MASS}(y) / \operatorname{NORM}(y)-\alpha$. Property $5.1(\mathrm{c}),(\mathrm{d})$ imply that $\alpha<(2+o(1)) \cdot \lambda_{j}$ and that DEG(y) $\leq 2 \alpha / \lambda_{j-1}+2$, where the +2 represents the stunted child and the child $z$ exempted from Property 5.1(d):

$$
\begin{aligned}
\operatorname{DEG}(y) \log \frac{\operatorname{MASS}(y)}{\operatorname{NORM}(y)} & \left.\leq\left(\frac{2 \alpha}{\lambda_{j-1}}+2\right) \log (\alpha+\beta) \quad \text { \{see explanations below }\right\} \\
& =O\left(\frac{\max \left\{\alpha \log \left(2 \lambda_{j}\right), \beta\right\}}{\lambda_{j-1}}\right) \\
& =O\left(\frac{\alpha+\beta}{2^{j-1}}\right)=O\left(\frac{\operatorname{MASS}(y)}{\operatorname{NORM}(y) \cdot 2^{j-1}}\right) .
\end{aligned}
$$

The first line follows from our bound on $\operatorname{DEG}(y)$ and the definitions of $\alpha$ and $\beta$. The second line follows since $\alpha<(2+o(1)) \lambda_{j}$ and $\alpha \log (\alpha+\beta)=O(\max \{\alpha \log \alpha, \beta\})$. The last line follows since $\log \lambda_{j}=\lambda_{j-1} / 2^{j-1}>1$. By the above bound and Property $5.1(\mathrm{e}), \sum_{y \in H(x)} \operatorname{DEG}(y) \log (\operatorname{MAsS}(y) / \operatorname{NORM}(y))=O(\operatorname{MASS}(x) / \operatorname{NORM}(x))$. Therefore, by Lemma 5.4, the second summations in both $\phi(\mathcal{H})$ and $\psi(\mathcal{H})$ are bounded by $O(n)$.

6. Limits of hierarchy-type algorithms. In this section we state a simple property (Property 6.1) of all hierarchy-type algorithms and give a lower bound on any undirected SSSP algorithm satisfying that property. The upshot is that our SSSP algorithm is optimal (up to an inverse-Ackermann factor) for a fairly large class of SSSP algorithms, which includes all hierarchy-type algorithms, variations on Dijkstra's algorithm, and even a heuristic SSSP algorithm [G01].

We will state Property 6.1 in terms of directed graphs. Let CYCLES $(u, v)$ denote the set of all cycles, including nonsimple cycles, that pass through both $u$ and $v$, and let $\operatorname{SEP}(u, v)=\min _{C \in \operatorname{CYCLES}(u, v)} \max _{e \in C} \ell(e)$. Note that in undirected graphs $\operatorname{SEP}(u, v)$ corresponds exactly to the longest edge on the MST path between $u$ and $v$.

PROPERTY 6.1. An SSSP algorithm with the hierarchy property computes, aside from shortest paths, a permutation $\pi_{s}: V(G) \rightarrow V(G)$ such that for any vertices $u, v$, we find $d(s, u) \geq d(s, v)+\operatorname{SEP}(u, v) \Longrightarrow \pi_{s}(u)>\pi_{s}(v)$, where $s$ is the source and $d$ the distance function.

The permutation $\pi_{s}$ corresponds to the order in which vertices are visited when the source is $s$. Property 6.1 says that $\pi_{s}$ is loosely sorted by distance, but may invert pairs of vertices if their relative distance is less than their SEP-value. To see that our hierarchy-based algorithm satisfies Property 6.1, consider two vertices $u$ and $v$. Let $x$ be the LCA of $u$ and $v$ in $\mathcal{H}$, and let $u^{\prime}$ and $v^{\prime}$ be the ancestors of $u$ and $v$, respectively, which are children of $x$. By our construction of $\mathcal{H}, \operatorname{NORM}(x) \leq \operatorname{seP}(u, v)$. If $d(s, u) \geq d(s, v)+\operatorname{SEP}(u, v)$, then $d(s, u) \geq d(s, v)+\operatorname{NORM}(x)$, and therefore the recursive calls on $u^{\prime}$ and $v^{\prime}$ that cause $u$ and $v$ to be visited are not passed the same interval argument, since both intervals have width $\operatorname{NORM}(x)$. The recursive call on $u^{\prime}$ 


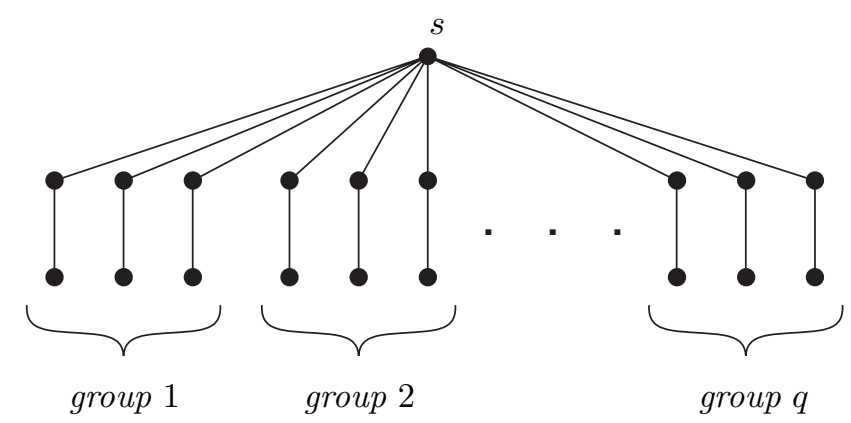

FIG. 4. The minimum spanning tree of the graph.

must, therefore, precede the recursive call on $v^{\prime}$, and $u$ must be visited before $v$.

THEOREM 6.1. Suppose that our computational model allows any set of functions from $\mathbb{R}^{O(1)} \rightarrow \mathbb{R}$ and comparison between two reals. Any SSSP algorithm for real-weighted graphs satisfying Property 6.1 makes $\Omega(m+\min \{n \log \log r, n \log n\})$ operations in the worst case, where $r$ is the ratio of the maximum to minimum edge length.

Proof. Let $q$ be an integer. Assume without loss of generality that $2 q$ divides $n-1$. The MST of the input graph is as depicted in Figure 4. It consists of the source vertex $s$, which is connected to $p=(n-1) / 2$ vertices in the top row, each of which is paired with one vertex in the bottom row. All the vertices (except $s$ ) are divided into disjoint groups, where group $i$ consists of exactly $p / q$ randomly chosen pairs of vertices. There are exactly $p ! /(p / q) !^{q}=q^{\Omega(p)}$ possible group arrangements. We will show that any algorithm satisfying Property 6.1 must be able to distinguish them.

We choose edge lengths as follows. All edges in group $i$ have length $2^{i}$. This includes edges from $s$ to the group's top row and between the two rows. Other non-MST edges are chosen so that shortest paths from $s$ correspond to paths in the MST. Let $v_{i}$ denote any vertex in the bottom row of group $i$. Then $d\left(s, v_{i}\right)=$ $2 \cdot 2^{i}$ and $\operatorname{SEP}\left(v_{i}, v_{j}\right)=2^{\max \{i, j\}}$. By Property $6.1, v_{i}$ must be visited before $v_{j}$ if $d\left(s, v_{i}\right)+\operatorname{SEP}\left(v_{i}, v_{j}\right) \leq d\left(s, v_{j}\right)$, which is true for $i<j$ since $2 \cdot 2^{i}+2^{j} \leq 2 \cdot 2^{j}$. Therefore, any algorithm satisfying Property 6.1 must be prepared to visit vertices in $q^{\Omega(p)}$ distinct permutations and make at least $\Omega(p \log q)=\Omega(n \log \log r)$ comparisons in the worst case. It also must include every non-MST edge in at least one operation, which gives the lower bound.

Theorem 6.1 shows that our SSSP algorithm is optimal among hierarchy-type algorithms, to within a tiny inverse-Ackermann factor. A lower bound on directed SSSP algorithms satisfying Property 6.1 is given in [Pet04]. Theorem 6.1 differs from that lower bound in two respects. First, the [Pet04] bound is $\Omega(m+\min \{n \log r, n \log n\})$, which is $\Omega(m+n \log n)$ for even reasonably small values of $r$. Second, the [Pet04] bound holds even if the algorithm is allowed to compute the SEP function (and sort the values) for free. Contrast this with our SSSP algorithm, where the main obstacle to achieving linear time is the need to sort the SEP-values.

7. Discussion. We have shown that with a near-linear time investment in preprocessing, SSSP queries can be answered in very close to linear time. Furthermore, among a natural class of SSSP algorithms captured by Property 5.1, our SSSP algorithm is optimal, aside from a tiny inverse-Ackermann factor. We can imagine several 
avenues for further research, the most interesting of which is developing a feasible alternative to Property 5.1 that does not have an intrinsic sorting bottleneck. This would be a backward approach to algorithm design: first we define a desirable property, then we hunt about for algorithms with that property. Another avenue, which might have some real-world impact, is to reduce the preprocessing cost of the directed shortest path algorithm in [Pet04] from $O(m n)$ to near-linear, as it is in our algorithm.

The marginal cost of computing SSSP with our algorithm may or may not be linear; it all depends on the complexity of the split-findmin structure. This data structure, invented first by Gabow [G85a] for use in a weighted matching algorithm, actually has connections with other fundamental problems. For instance, it can be used to solve both the minimum spanning tree and shortest path tree sensitivity analysis problems [Pet03]. (The sensitivity analysis problem is to decide how much each edge's length can be perturbed without changing the solution tree.) Therefore, by Theorem 4.2 both these problems have complexity $O(m \log \alpha(m, n))$, an $\alpha / \log \alpha$ improvement over Tarjan's path-compression-based algorithm [Tar82]. If we consider the offline version of the split-findmin problem, where all splits and decrease-keys are given in advance, one can show that it is reducible to both the MST problem and the MST sensitivity analysis problem. None of these reductions proves whether $\operatorname{MST}(m, n)$ dominates $\operatorname{SPLIT-FINDMIN}(m, n)$ or vice versa; however, they do suggest that we have no hope of solving the MST problem [PR02b, PR02c] without first solving the manifestly simpler split-findmin and MST sensitivity analysis problems.

The experimental study of Pettie, Ramachandran, and Sridhar [PRS02] shows that our algorithm is very efficient in practice. However, the [PRS02] study did not explore all possible implementation choices, such as the proper heap to use, the best preprocessing algorithm, or different implementations of the split-findmin structure. To our knowledge no one has investigated whether the other hierarchy-type algorithms [Tho99, Hag00, Pet04] are competitive in real-world scenarios.

An outstanding research problem in parallel computing is to bound the time-work complexity of SSSP. There are several published algorithms on the subject [BTZ98, CMMS98, KS97, M02, TZ96], though none runs in worst-case polylogarithmic time using work comparable to Dijkstra's algorithm. There is clearly a lot of parallelism in the hierarchy-based algorithms. Whether this approach can be effectively parallelized is an intriguing question.

Appendix A. The bucket-heap. The bucket-heap structure consists of an array of buckets, where the $i$ th bucket spans the interval $[\delta+i \mu, \delta+(i+1) \mu)$, for fixed reals $\delta$ and $\mu$. Logically speaking, a heap item with key $\kappa$ appears in the bucket whose interval spans $\kappa$. We are never concerned about the relative order of items within the same bucket.

Proof of Lemma 4.3. Our structure simulates the logical specification given earler; it actually consists of levels of bucket arrays. The level zero buckets are the ones referred to in the bucket-heap's specification, and the level $i$ buckets preside over disjoint intervals of $2^{i}$ level zero buckets. The interval represented by a higher-level bucket is the union of its component level zero buckets. Only one bucket at each level is active: it is the first one that presides over no closed level zero buckets; see Figure 5. Suppose that an item $x$ should logically be in the level zero bucket $B$. We maintain the invariant that $x$ is either descending and in the lowest active bucket presiding over $B$, or ascending and in some active bucket presiding over level zero buckets before $B$.

To insert a node we put it in the first open level zero bucket and label it as ascending. This clearly satisfies the invariant. The result of a decrease-key depends 

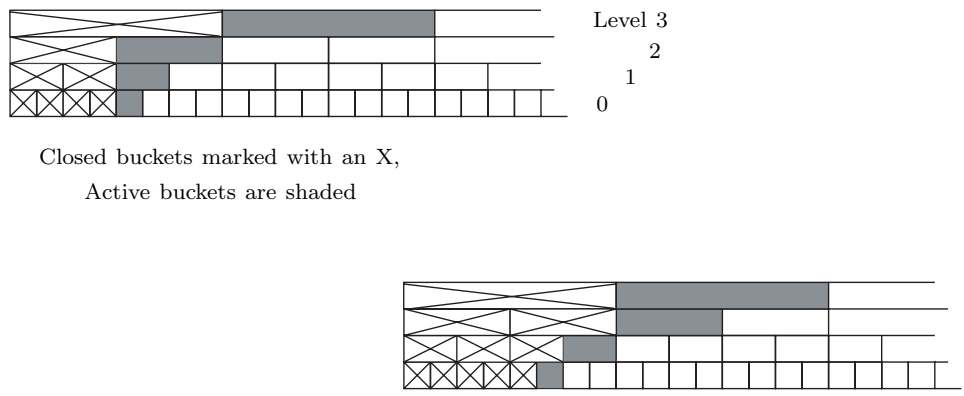

The effect of closing the first open bucket

FIG. 5. Active buckets are shaded.

on whether the node $x$ is ascending or descending. Suppose $x$ is ascending and in a bucket (at some level) spanning the interval $[a, b)$. If $\operatorname{key}(x)<b$, we relabel it as descending; otherwise we do nothing. If $x$ is descending (or was just relabeled as descending), we move it to the lowest level active bucket consistent with the invariant. If $x$ drops $i \geq 0$ levels, we assume that this is accomplished in $O(i+1)$ time; i.e., we search from its current level down, not from the bottom up.

Suppose that we close the first open level zero bucket $B$. According to the invariant all items that are logically in $B$ are descending and actually in $B$, so enumerating them is no problem; there will, in general, be ascending items in $B$ that do not logically belong there. In order to maintain the invariant we must deactivate all active buckets that preside over $B$ (including $B$ ). Consider one such bucket at level $i$. If $i>0$, we move each descending node in it to the level $i-1$ active bucket. For each ascending node (at level $i \geq 0$ ), depending on its key, we either move it to the level $i+1$ active bucket and keep it ascending, or relabel it descending and move it to the proper active bucket at level $\leq i+1$.

From the invariant it follows that no node $x$ appears in more than $2 \log \Delta_{x}+1$ distinct buckets: $\log \Delta_{x}+1$ buckets as an ascending node and another $\log \Delta_{x}$ as a descending node. Aside from this cost of moving nodes around, the other costs are clearly $O(N)$.

We remark that the bucket-heap need not actually label the items. Whether an item is ascending or descending can be inferred from context.

Appendix B. The split-findmin problem. The split-findmin problem is to maintain a collection of sequences of weighted elements under the following operations:

$$
\begin{aligned}
\operatorname{split}(x): & \text { Split the sequence containing } x \text { into two sequences: the } \\
& \text { elements up to and including } x \text { and the rest. } \\
\text { decrease-key }(x, \kappa): & \text { Set } \operatorname{key}(x)=\min \{\operatorname{key}(x), \kappa\} .
\end{aligned}
$$

Gabow [G85a] gave an elegant algorithm for this problem that is nearly optimal. On an initial sequence of $n$ elements, it handles up to $n-1$ splits and $m$ decrease-keys in $O((m+n) \alpha(m, n))$ time. Gabow's algorithm runs on a pointer machine [Tar79]. We now prove Theorem 4.2 from section 4.2.

Proof of Theorem 4.2. In Gabow's decrease-key routine a sequence of roughly $\alpha$ variables needs to be updated, although it is already known that their values are 
monotonically decreasing. We observe that, on a pointer machine, the same task can be accomplished in $O(\alpha)$ time using $O(\log \alpha)$ comparisons for a binary search. Using a simple two-level scheme, one can easily reduce the $n \alpha$ term in the running time to $n$. This gives the split-findmin algorithm that performs $O(m \log \alpha(m, n)+n)$ comparisons.

To get a potentially faster algorithm on the RAM model we construct all possible split-findmin solvers on inputs with at most $q=\log \log n$ elements and choose one that is close to optimal for all problem sizes. We then show how to compose this optimal split-findmin solver on $q$ elements with Gabow's structure to get an optimal solver on $n$ elements.

We consider only instances with $m^{\prime}<q^{2}$ decrease-keys. If more decrease-keys are actually encountered, we can revert to Gabow's algorithm [G85a] or a trivial one that runs in $O\left(m^{\prime}\right)$ time.

We represent the state of the solver with three components: a bit-vector with length $q-1$, representing where the splits are; a directed graph $H$ on no more than $q+m^{\prime}<q(q+1)$ vertices, representing known inequalities between current keys and older keys retired by decrease-key operations; and finally, a mapping from elements to vertices in $H$. One may easily confirm that the state can be represented in no more than $3 q^{4}=o(\log n)$ bits. One may also confirm that a split or decrease-key can update the state in $O(1)$ time. We now turn to the findmin operation. Consider the findmin-action function, which determines the next step in the findmin procedure. It can be represented as

$$
\text { findmin-action : state } \times\{1, \ldots, q\} \rightarrow(V(H) \times V(H)) \cup\{1, \ldots, q\},
$$

where the first $\{1, \ldots, q\}$ represents the argument to the findmin query. The findminaction function can either perform a comparison (represented by $V(H) \times V(H)$ ) which, if performed, will alter the state, or return an answer to the findmin query, represented by the second $\{1, \ldots, q\}$. One simply applies the findmin-action function until it produces an answer. We will represent the findmin-action function as a table. Since the state is represented in $o(\log n)$ bits, we can keep it in one machine word; therefore, computing the findmin-action function (and updating the state) takes constant time on a RAM.

One can see that any split-findmin solver can be converted, without loss of efficiency, into one that performs comparisons only during calls to findmin. Therefore, finding the optimal findmin-action function is tantamount to finding the optimal splitfindmin solver.

We have now reduced the split-findmin problem to a brute force search over the findmin-action function. There are less than $F=2^{3 q^{4}} \cdot q \cdot\left(q^{4}+q\right)<2^{4 q^{4}}$ distinct findmin-action functions, most of which do not produce correct answers. There are less than $I=\left(2 q+q^{2}(q+1)\right)^{q^{2}+3 q}$ distinct instances of the problem, because the number of decrease-keys is $<q^{2}$, findmins $<2 q$, and splits $<q$. Furthermore, each operation can be a split or findmin, giving the $2 q$ term, or a decrease-key, which requires us to choose an element and where to fit its new key into the permutation, giving the $q^{2}(q+1)$ term. Each findmin-action/problem instance pair can be tested for correctness in $V=O\left(q^{2}\right)$ time, and therefore all correct findmin-action functions can be chosen in time $F \cdot I \cdot V=2^{\Omega\left(q^{4}\right)}$. For $q=\log \log n$ this is $o(n)$, meaning the time for this brute force search does not affect the other constant factors involved.

How do we choose the optimal split-findmin solver? This is actually not a trivial question because of the possibility of there not being one solver that dominates all 
others on all input sizes. Consider charting the worst-case complexity of a solver $\mathcal{S}$ as a function $g_{\mathcal{S}}$ of the number of operations $p$ in the input sequence. It is plausible that certain solvers are optimal for only certain densities $p / q$. We need to show that for some solver $\mathcal{S}^{*}, g_{\mathcal{S}^{*}}$ is within a constant factor of the lower envelope of $\left\{g_{\mathcal{S}}\right\}_{\mathcal{S}}$, where $\mathcal{S}$ ranges over all correct solvers. Let $\mathcal{S}_{k}$ be the optimal solver for $2^{k}$ operations. We let $\mathcal{S}^{*}$ be the solver that mimics $\mathcal{S}_{k}$ from operations $2^{k-1}+1$ to $2^{k}$. At operation $2^{k}$ it resets its state, reexecutes all $2^{k}$ operations under $\mathcal{S}_{k+1}$, and continues using $\mathcal{S}_{k+1}$ until operation $2^{k+1}$. Since $g_{\mathcal{S}_{k+1}}\left(2^{k+1}\right) \leq 2 \cdot g_{\mathcal{S}_{k}}\left(2^{k}\right)$, it follows that $g_{\mathcal{S}^{*}}(p) \leq 4 \cdot \min _{\mathcal{S}}\left\{g_{\mathcal{S}}(p)\right\}$.

Our overall algorithm is very simple. We divide the $n$ elements into $n^{\prime}=n / q$ superelements, each representing a contiguous block of $q$ elements. Each unsplit sequence then consists of three parts: two subsequences in the leftmost and rightmost superelements and a third subsequence consisting of unsplit superelements. We use Gabow's algorithm on the unsplit superelements, where the key of a superelement is the minimum over constituent elements. For the superelements already split, we use the $\mathcal{S}^{*}$ split-findmin solver constructed as above. The cost of Gabow's algorithm is $O((m+n / q) \alpha(m, n / q))=O(m+n)$, and the cost of using $\mathcal{S}^{*}$ on each superelement is $\Theta(\operatorname{SPLIT-FINDMIN}(m, n))$ by construction; therefore the overall cost is $\Theta(\operatorname{sPLIT-Findmin}(m, n))$.

One can easily extend the proof to randomized split-findmin solvers by defining the findmin-action as selecting a distribution over actions.

We note that the time bound of Theorem 4.2 on pointer machines is provably optimal. La Poutré [LaP96] gave a lower bound on the pointer machine complexity of the split-find problem, which is subsumed by the split-findmin problem. The results in this section address the RAM complexity and decision-tree complexity of splitfindmin, which are unrelated to La Poutré's result.

\section{REFERENCES}

[AGM97] N. Alon, Z. Galil, And O. Margalit, On the exponent of the all pairs shortest path problem, J. Comput. System Sci., 54 (1997), pp. 255-262.

[AHU76] A. V. Aho, J. E. Hopcroft, And J. D. Ullman, On finding lowest common ancestors in trees, SIAM J. Comput., 5 (1976), pp. 115-132.

[Am093] R. K. Ahuja, T. L. Magnati, And J. B. Orlin, Network Flows: Theory, Algorithms, and Applications, Prentice-Hall, Englewood Cliffs, NJ, 1993.

[AmOt90] R. K. Ahuja, K. Mehlhorn, J. B. Orlin, and R. E. TARjan, Faster algorithms for the shortest path problem, J. ACM, 37 (1990), pp. 213-223.

[BKRW98] A. L. Buchsbaum, H. Kaplan, A. Rogers, and J. R. Westbrook, Linear-time pointer-machine algorithms for LCAs, MST verification, and dominators, in Proceedings of the 30th ACM Symposium on Theory of Computing (STOC), Dallas, TX, 1998, ACM, New York, 1998, pp. 279-288.

[BTZ98] G. S. Brodal, J. L. TräfF, and C. D. Zaroliagis, A parallel priority queue with constant time operations, J. Parallel and Distrib. Comput., 49 (1998), pp. 4-21.

[Chaz00] B. Chazelle, A minimum spanning tree algorithm with inverse-Ackermann type complexity, J. ACM, 47 (2000), pp. 1028-1047.

[CLRS01] T. H. Cormen, C. E. Leiserson, R. L. Rivest, and C. Stein, Introduction to Algorithms, MIT Press, Cambridge, MA, 2001.

[CMms98] A. Crauser, K. Mehlhorn, U. Meyer, and P. Sanders, A parallelization of Dijkstra's shortest path algorithm, in Proceedings of the 23rd International Symposium on Mathematical Foundations of Computer Science (MFCS), Lecture Notes in Comput. Sci. 1450, Springer, New York, 1998, pp. 722-731.

[Dij59] E. W. Dijkstra, A note on two problems in connexion with graphs, Numer. Math., 1 (1959), pp. 269-271.

[Din78] E. A. DinIC, Economical algorithms for finding shortest paths in a network, Transportation Modeling Systems, (1978), pp. 36-44 (in Russian). 
[Din03] Y. Dinitz, Personal communication, Ben-Gurion University, Be'er Sheva, Israel, 2003.

[FG85] A. M. FRIEZE AND G. R. GRIMmetT, The shortest-path problem for graphs with random arc-lengths, Discrete Appl. Math., 10 (1985), pp. 57-77.

[FR01] J. FAKChaROENPHOL AND S. RAO, Planar graphs, negative weight edges, shortest paths, and near linear time, in Proceedings of the 42nd IEEE Symposium on Foundations of Computer Science (FOCS), Las Vegas, NV, 2001, IEEE Press, Piscataway, NJ, pp. 232-241.

[F91] G. N. Frederickson, Planar graph decomposition and all pairs shortest paths, J. ACM, 38 (1991), pp. 162-204.

[F76] M. L. Fredman, New bounds on the complexity of the shortest path problem, SIAM J. Comput., 5 (1976), pp. 83-89.

[FT87] M. L. Fredman AND R. E. TARJAN, Fibonacci heaps and their uses in improved network optimization algorithms, J. ACM, 34 (1987), pp. 596-615.

[FW93] M. L. Fredman AND D. E. Willard, Surpassing the information-theoretic bound with fusion trees, J. Comput. System Sci., 47 (1993), pp. 424-436.

[G01] A. V. GoldBerg, A simple shortest path algorithm with linear average time, in Proceedings of the 9th European Symposium on Algorithms (ESA), Lecture Notes in Comput. Sci. 2161, Springer, New York, 2001, pp. 230-241.

[G85a] H. N. GABOw, A scaling algorithm for weighted matching on general graphs, in Proceedings of the 26th IEEE Symposium on Foundations of Computer Science (FOCS), Portland, OR, 1985, IEEE Press, Piscataway, NJ, pp. 90-100.

[G85b] H. N. Gabow, Scaling algorithms for network problems, J. Comput. System Sci., 31 (1985), pp. 148-168.

[G95] A. V. GoldBerg, Scaling algorithms for the shortest paths problem, SIAM J. Comput., 24 (1995), pp. 494-504.

[GM97] Z. Galil and O. Margalit, All pairs shortest distances for graphs with small integer length edges, Inform. and Comput., 134 (1997), pp. 103-139.

[GR98] A. V. GoldBerg And S. RAO, Beyond the flow decomposition barrier, J. ACM, 45 (1998), pp. 783-797.

[GT89] H. N. Gabow AND R. E. TARJAn, Faster scaling algorithms for network problems, SIAM J. Comput., 18 (1989), pp. 1013-1036.

[GT91] H. N. GABOW AND R. E. TARJAN, Faster scaling algorithms for general graph-matching problems, J. ACM, 38 (1991), pp. 815-853.

[GYY80] R. L. Graham, A. C. YAO, AND F. F. YAO, Information bounds are weak in the shortest distance problem, J. ACM, 27 (1980), pp. 428-444.

[Hag00] T. Hagerup, Improved shortest paths on the word RAM, in Proceedings of the 27th International Colloquium on Automata, Languages, and Programming (ICALP), Lecture Notes in Comput. Sci. 1853, Springer, New York, 2000, pp. 61-72.

[Hag04] T. HAGERUP, Simpler computation of single-source shortest paths in linear average time, in Proceedings in the 21st Annual Symposium on Theoretical Aspects of Computer Science (STACS), Montpellier, France, 2004, Springer, New York, pp. 362369.

[Han04] Y. HAN, Improved algorithm for all pairs shortest paths, Inform. Process. Lett., 91 (2004), pp. 245-250.

[HKRs97] M. R. Henzinger, P. N. Klein, S. Rao, and S. Subramanian, Faster shortest path algorithms for planar graphs, J. Comput. System Sci., 55 (1997), pp. 3-23.

[HT02] Y. HAN AND M. THORUP, Integer sorting in $O(n \sqrt{\log \log n})$ expected time and linear space, in Proceedings of the 43rd Annual Symposium on Foundations of Computer Science (FOCS), Vancouver, 2002, IEEE Press, Piscataway, NJ, pp. 135-144.

[J77] D. B. Johnson, Efficient algorithms for shortest paths in sparse networks, J. ACM, 24 (1977), pp. 1-13.

[K70] L. R. KERR, The Effect of Algebraic Structure on the Computational Complexity of Matrix Multiplication, Technical report TR70-75, Computer Science Department, Cornell University, Ithaca, NY, 1970.

[KKP93] D. R. Karger, D. Koller, and S. J. Phillips, Finding the hidden path: Time bounds for all-pairs shortest paths, SIAM J. Comput., 22 (1993), pp. 1199-1217.

[KKT95] D. R. Karger, P. N. Klein, And R. E. TARJAn, A randomized linear-time algorithm for finding minimum spanning trees, J. ACM, 42 (1995), pp. 321-329.

[KS97] P. N. Klein And S. Subramanian, A randomized parallel algorithm for single-source shortest paths, J. Algorithms, 25 (1997), pp. 205-220.

[KS98] S. G. Kolliopoulos AND C. Stein, Finding real-valued single-source shortest paths in $o\left(n^{3}\right)$ expected time, J. Algorithms, 28 (1998), pp. 125-141. 
[LaP96] H. LAPoutRÉ, Lower bounds for the union-find and the split-find problem on pointer machines, J. Comput. System Sci., 52 (1996), pp. 87-99.

[M01] U. MEYER, Single-source shortest-paths on arbitrary directed graphs in linear averagecase time, in Proceedings of the 12th Annual ACM-SIAM Symposium on Discrete Algorithms (SODA), Washington, DC, 2001, SIAM, Philadelphia, pp. 797-806.

[M02] U. MEYER, Buckets strike back: Improved parallel shortest-paths, in Proceedings of the 16th International Parallel and Distributed Processing Symposium (IPDPS), Ft. Lauderdale, FL, 2002, IEEE Computer Society Press, Los Alamitos, CA, pp. 7582.

[Mit00] J. S. B. Mitchell, Geometric shortest paths and network optimization, in Handbook of Computational Geometry, North-Holland, Amsterdam, 2000, pp. 633-701.

[MN00] K. Mehlhorn And S. NÄher, LEDA: A Platform for Combinatorial and Geometric Computing, Cambridge University Press, Cambridge, UK, 2000.

[MT87] A. MOFFAT AND T. TAKAOKA, An all pairs shortest path algorithm with expected time $O\left(n^{2} \log n\right)$, SIAM J. Comput., 16 (1987), pp. 1023-1031.

[Pet02b] S. PeTtie, On the comparison-addition complexity of all-pairs shortest paths, in Proceedings of the 13th International Symposium on Algorithms and Computation (ISAAC'02), Vancouver, 2002, Springer, New York, pp. 32-43.

[Pet03] S. Pettie, On the Shortest Path and Minimum Spanning Tree Problems, Ph.D. thesis, Department of Computer Sciences, The University of Texas at Austin, Austin, TX, 2003; also available online as Technical report TR-03-35 at http://www.cs.utexas.edu/ftp/pub/techreports/tr03-35.ps.gz.

[Pet04] S. PeTtie, A new approach to all-pairs shortest paths on real-weighted graphs, Special Issue of Selected Papers from the 29th International Colloqium on Automata Languages and Programming (ICALP 2002), Theoret. Comput. Sci., 312 (2004), pp. 47-74.

[PR02a] S. Pettie And V. Ramachandran, Computing shortest paths with comparisons and additions, in Proceedings of the 13th Annual ACM-SIAM Symposium on Discrete Algorithms (SODA), San Francisco, CA, 2002, SIAM, Philadelphia, pp. 267-276.

[PR02b] S. Pettie And V. Ramachandran, Minimizing randomness in minimum spanning tree, parallel connectivity, and set maxima algorithms, in Proceedings of the 13th Annual ACM-SIAM Symposium on Discrete Algorithms (SODA), San Francisco, CA, 2002, SIAM, Philadelphia, pp. 713-722.

[PR02c] S. PetTiE And V. RAMAChandRAn, An optimal minimum spanning tree algorithm, J. ACM, 49 (2002), pp. 16-34.

[PRS02] S. Pettie, V. Ramachandran, And S. SRIdhar, Experimental evaluation of a new shortest path algorithm, in Proceedings of the 4th Workshop on Algorithm Engineering and Experiments (ALENEX), San Francisco, CA, 2002, Springer, New York, pp. 126-142.

[Sei95] R. SEIDEL, On the all-pairs-shortest-path problem in unweighted undirected graphs, J. Comput. System Sci., 51 (1995), pp. 400-403.

[SP75] P. M. SPIRA AND A. PAN, On finding and updating spanning trees and shortest paths, SIAM J. Comput., 4 (1975), pp. 375-380.

[Spi73] P. M. SPIRA, A new algorithm for finding all shortest paths in a graph of positive arcs in average time $O\left(n^{2} \log ^{2} n\right)$, SIAM J. Comput., 2 (1973), pp. 28-32.

[SZ99] A. Shoshan And U. ZwICK, All pairs shortest paths in undirected graphs with integer weights, in Proceedings of the 40th Annual IEEE Symposium on Foundations of Computer Science (FOCS), New York, 1999, IEEE Press, Piscataway, NJ, pp. 605614 .

[Tak92] T. TAKAOKA, A new upper bound on the complexity of the all pairs shortest path problem, Inform. Process. Lett., 43 (1992), pp. 195-199.

[Tak98] T. TAKAOKA, Subcubic cost algorithms for the all pairs shortest path problem, Algorithmica, 20 (1998), pp. 309-318.

[Tar79] R. E. TARJAN, A class of algorithms which require nonlinear time to maintain disjoint sets, J. Comput. System Sci., 18 (1979), pp. 110-127.

[Tar79b] R. E. TARJAN, Applications of path compression on balanced trees, J. ACM, 26 (1979), pp. 690-715.

[Tar82] R. E. TARJAN, Sensitivity analysis of minimum spanning trees and shortest path trees, Inform. Process. Lett., 14 (1982), pp. 30-33; Corrigendum, Inform. Process. Lett., 23 (1986), p. 219.

[Tho00] M. Thorup, Floats, integers, and single source shortest paths, J. Algorithms, 35 (2000), pp. 189-201. 
[Tho03] M. Thorup, Integer priority queues with decrease key in constant time and the single source shortest paths problem, in Proceedings of the 35th Annual ACM Symposium on Theory of Computing (STOC), San Diego, CA, 2003, ACM, New York, pp. 149158.

[Tho99] M. THORUP, Undirected single-source shortest paths with positive integer weights in linear time, J. ACM, 46 (1999), pp. 362-394.

[TZ96] J. L. TRÄFF AND C. D. ZAROliagis, A simple parallel algorithm for the single-source shortest path problem on planar digraphs, in Parallel Algorithms for Irregularly Structured Problems, Lecture Notes in Comput. Sci. 1117, Springer, New York, 1996, pp. 183-194.

[Z01] U. ZwICK, Exact and approximate distances in graphs - A survey, in Proceedings of the 9th European Symposium on Algorithms (ESA), University of Aarhus, Denmark, 2001, pp. 33-48; available online at http://www.cs.tau.ac.il/ zwick/.

[Z02] U. ZwICK, All pairs shortest paths using bridging sets and rectangular matrix multiplication, J. ACM, 49 (2002), pp. 289-317.

[Z04] U. ZwICK, A slightly improved sub-cubic algorithm for the all pairs shortest paths problem with real edge lengths, in Proceedings of the 15th International Symposium on Algorithms and Computation (ISAAC), Lecture Notes in Comput. Sci. 3341, Springer, New York, 2004, pp. 921-932. 